\title{
Specification of Eddy Transfer Coefficients in Coarse-Resolution Ocean Circulation Models*
}

\author{
Martin Visbeck, ${ }^{+}$John Marshall, And Tom Haine \\ Center for Meteorology and Physical Oceanography, Department of Earth, Atmospheric, and Planetary Science, \\ Massachusetts Institute of Technology, Cambridge, Massachusetts \\ MiKe SPALl \\ Department of Physical Oceanography, Woods Hole Oceanographic Institution, Woods Hole, Massachusetts
}

(Manuscript received 8 December 1995, in final form 2 July 1996)

\begin{abstract}
Parametric representations of oceanic geostrophic eddy transfer of heat and salt are studied ranging from horizontal diffusion to the more physically based approaches of Green and Stone (GS) and Gent and McWilliams (GM). The authors argue for a representation that combines the best aspects of GS and GM: transfer coefficients that vary in space and time in a manner that depends on the large-scale density fields (GS) and adoption of a transformed Eulerian mean formalism (GM). Recommendations are based upon a two-dimensional (zonally or azimuthally averaged) model with parameterized horizontal and vertical fluxes that is compared to three-dimensional numerical calculations in which the eddy transfer is resolved. Three different scenarios are considered: 1) a convective "chimney" where the baroclinic zone is created by differential surface cooling; 2) spindown of a frontal zone due to baroclinic eddies; and 3) a wind-driven, baroclinically unstable channel. Guided by baroclinic instability theory and calibrated against eddy-resolving calculations, the authors recommend a form for the horizontal transfer coefficient given by

$$
k=\alpha \frac{f}{\sqrt{\mathrm{Ri}}} l^{2}=\alpha \frac{M^{2}}{N} l^{2},
$$

where $\mathrm{Ri}=f^{2} N^{2} / M^{4}$ is the large-scale Richardson number and $f$ is the Coriolis parameter; $M^{2}$ and $N^{2}$ are measures of the horizontal and vertical stratification of the large-scale flow, $l$ measures the width of the baroclinic zone, and $\alpha$ is a constant of proportionality. In the very different scenarios studied here the authors find $\alpha$ to be a "universal" constant equal to 0.015 , not dissimilar to that found by Green for geostrophic eddies in the atmosphere. The magnitude of the implied $k$, however, varies from $300 \mathrm{~m}^{2} \mathrm{~s}^{-1}$ in the chimney to $2000 \mathrm{~m}^{2} \mathrm{~s}^{-1}$ in the wind-driven channel.
\end{abstract}

\section{Introduction}

Large-scale models used to study and simulate the general circulation of the ocean must parameterize the important transfer properties of unresolved baroclinic eddies. Because the ratio of the dominant energy-containing scale in the ocean to the scale of ocean basins is so large and despite phenomenal increases in com-

\footnotetext{
* Contribution Number 5496 from Lamont-Doherty Earth Observatory and Contribution Number 9154 from Woods Hole Oceanographic Institution.

${ }^{+}$Current affiliation: Lamont-Doherty Earth Observatory of Columbia University, Palisades, NY.
}

Corresponding author address: Dr. Martin Visbeck, Lamont-Doherty Earth Observatory, Columbia University, Palisades, NY 10964. E-mail: visbeck@1deo.columbia.edu puter power over the past two decades, global ocean models that resolve the geostrophic eddy field can only be integrated for a few tens of years, even when the biggest and fastest computers available today are employed. The practical need to improve the parametric representation of geostrophic eddies for the study of the ocean on climatic timescales thus remains as urgent as ever. The intellectual need also remains, for one would hope that a model of the climate could be constructed in which the detail of geostrophic eddies in the ocean (and perhaps also even in the atmosphere) need not be resolved.

Despite the acknowledged importance of the geostrophic eddy field in the ocean, its representation in large-scale ocean models is rather rudimentary; essentially ocean climate models assume that heat, salt, and momentum are "diffused" down the large-scale gradient. For example, the eddy flux of heat is related to mean quantities; thus, 


$$
\overline{v^{\prime} \tau^{\prime}}=-k \nabla_{h} \bar{\tau}
$$

where $k$ is set to an a priori value constant both in space and in time. Analogous expressions are used for salinity and, more problematically, also to momentum. Equation (1) with $k$ held constant is widely assumed in largescale ocean models; a constant $k$ is also assumed in the transformed Eulerian mean formulation recently advocated by Gent and McWilliams (1990) and Gent et al. (1995). There are many questions raised by (1): Is such a form reasonable? How large should the $k$ be? How do they vary in space and time?

In this paper we accept the supposition (1) for conserved, and hence transferable, quantities such as $T$ and $S$, but with two caveats: (i) Following Green (1970) and Stone (1972) we will suppose that the transfer occurs not in a horizontal plane but in a plane inclined at some angle to the horizontal and (ii) we regard Eq. (1) as a parameterization of the divergent, rather than the total eddy flux [see Marshall and Shutts (1981), where this interpretation of (1) is argued on the basis of the temperature variance equation]. The form (1) is not, however, appropriate as a representation of momentum transfer (e.g., $\overline{v^{\prime} u^{\prime}} \neq-k \partial \bar{u} / \partial y$ ) because baroclinic eddies can, and often do, transfer momentum up the local momentum gradient. As argued in Green (1970) and Marshall (1981), momentum is not conserved by a particle of fluid (it is continually being changed by pressure gradient forces), and the momentum transferring properties of baroclinic eddies is not parameterizable as a momentum diffusion.

In the present paper we do not attempt to develop a general parameterization of the heat and momentum transferring properties of the oceanic baroclinic eddy field. We believe that any such attempt must focus on the eddy flux of potential vorticity (PV) (Green 1970; Marshall 1981; Rhines and Young 1982). Our scope is much less ambitious. Here we confine our attention to the parameterization of heat (and salt) alone and thereby only address the large-scale PV fields. For large-scale motions PV is dominated by the planetary vorticity and stretching contributions, and relative vorticity contributions are generally smaller. We will apply the ideas of Green (1970) to deduce the likely manner in which the $k$ in (1) vary in space and time.

Green (1970)_-see also Stone (1972)—drew on insights from linear baroclinic instability analysis and the energetics of quasigeostrophic motion and developed a zonally averaged model of the atmosphere in which the heat and potential vorticity transport by baroclinic eddies was parameterized in terms of a transfer coefficient $k$ of the form:

$$
k=\alpha \frac{f}{\sqrt{\mathrm{Ri}}} l^{2}=\alpha \frac{M^{2}}{N} l^{2},
$$

where $f$ is the local Coriolis parameter, $l$ is a measure of the meridional distance over which particles are transferred, $\mathrm{Ri}$ is the Richardson number of the large-scale flow, and $\alpha$ is a constant of proportionality. Recognizing that $f / \sqrt{\mathrm{Ri}}$ is a measure of the growth rate of an Eady wave (Eady 1949), Eq. (2) makes good physical sense setting $k$ equal to an eddy velocity

$$
v_{\text {eddy }}=\frac{f}{\sqrt{\mathrm{Ri}}} l
$$

times a length scale $l$.

The authors have found that the energetic analysis of baroclinic zones leading to (2) gives, in the context of convectively driven baroclinically-unstable chimneys, both quantitative and parametric guidance to the depth to which convective chimneys can reach before deepening is arrested by baroclinic instability (see Visbeck et al. 1996; Haine and Marshall (1997, hereafter HM${ }^{1}$ ). We show here that use of a closure of the form (1), together with specification of the $k$ as in (2), leads to spatial variations in eddy fluxes that can capture the gross effect of the eddies on the large-scale flow. We evaluate the closure in the light of three different eddyresolving numerical simulations: 1) a convective "chimney" created by localized cooling (Visbeck et al. 1996), 2) an upper ocean front eroded due to baroclinic eddies (Spall 1995), and 3) a wind-forced stratified channel analogous to the Antarctic Circumpolar Current.

We compare zonally (or azimuthally) averaged fields from eddy-resolving models with those obtained from a parameterized two-dimensional model in the $y-z$ plane. The most satisfactory performance is found using a hybrid scheme that consists of the transformed Eulerian mean formalism (Gent and McWilliams 1990) combined with transfer coefficients that depend on the large-scale density field (2) (Green 1970).

\section{Parameterizing the transfer properties of baroclinic eddies}

Large-scale ocean models that do not have sufficient resolution to resolve the geostrophic eddy field must parameterize its transfer properties. Defining the "mean"-_enoted by $\bar{\tau}$ - as an Eulerian average over a period long compared to an eddy turnover time and the "eddy" - denoted by $\tau^{\prime}$ —as the departure from that mean, the "Reynold's-averaged" tracer equation can be written, using height as a vertical coordinate,

$$
\frac{\partial \bar{\tau}}{\partial t}+\overline{\mathbf{v}} \cdot \nabla_{h} \bar{\tau}+\bar{w} \frac{\partial \bar{\tau}}{\partial z}=\overline{S_{0}}-\nabla_{h} \cdot\left(\overline{\mathbf{v}^{\prime} \tau^{\prime}}\right)-\frac{\partial}{\partial z}\left(\overline{w^{\prime} \tau^{\prime}}\right) .
$$

Here $\mathbf{v}=(u, v)$ denotes the horizontal and $w$ the vertical velocity of the incompressible Boussinesq fluid. The tracer quantity $\tau$ could be the temperature, the salinity,

\footnotetext{
${ }^{1}$ Manuscript submitted to J. Phys. Oceanogr.
} 
a transient tracer, or an active tracer such as potential vorticity. The right-hand side of (3) contains sources and sinks $S_{0}$ together with the eddy flux divergence terms due to baroclinic eddies, which redistribute the tracer within the body of fluid. In coarse-resolution models these flux divergences must be expressed-parameterized-in terms of the averaged quantities carried explicitly in the model.

The simplest and most widely used parameterization is to suppose that the eddy transfer may be represented as a downgradient transfer (Fickian diffusion); thus,

$$
\overline{\mathbf{v}^{\prime} \tau^{\prime}}=-k^{\mathrm{hor}} \boldsymbol{\nabla}_{h} \bar{\tau}
$$

where the transfer coefficient $k^{\text {hor }}$ is chosen to be constant in time and space.

Mixing along the vertical axis is imagined to occur through the agency of internal waves (see Large et al. 1994, for a review) and is represented as

$$
\overline{w^{\prime} \tau^{\prime}}=-k^{\mathrm{ver}} \frac{\partial \bar{\tau}}{\partial z}
$$

\section{a. Eddy transfer theory: Green and Stone}

Green (1970) recognized that the transfer properties of baroclinic eddies in the troposphere of the atmosphere was a consequence of, and hence parameterizable in terms of, their structure. Supposing that transfer of heat occurs in the growing phase of baroclinic eddies, when energy is extracted by the eddy from the baroclinic zone of the large-scale flow, Green used energetic arguments to deduce the expected form of the transfer coefficients in (1) in the limit of large Richardson number. He used linear stability analysis to reveal the structure of the baroclinic wave and to guide his choice of the spatial form of the $k$. Stone (1972) derived a similar expression from linear stability theory and extended Green's results to the case when the Richardson number need not be large. Green and Stone (hereafter GS) imagined that diabatic processes then restore the baroclinic zone, replenishing the supply of available potential energy until it is "discharged" again by baroclinic instability. This repeated, and presumably intermittent, conversion of energy leads to an eddy flux that crosses the mean isentropic surfaces. Green and Stone attempted to relate the meridional and vertical eddy fluxes in zonally averaged atmospheric models to large-scale parameters; thus,

$$
\begin{aligned}
& \overline{v^{\prime} \tau^{\prime}}=-k_{v y} \frac{\partial \bar{\tau}}{\partial y}-k_{v z} \frac{\partial \bar{\tau}}{\partial z} \\
& \overline{w^{\prime} \tau^{\prime}}=-k_{w y} \frac{\partial \bar{\tau}}{\partial y}-k_{w z} \frac{\partial \bar{\tau}}{\partial z},
\end{aligned}
$$

where the transfer coefficients $k_{v y}, k_{v z}$ can be related to one another in terms of the isopycnal slope

$$
s_{\text {rho }}=\frac{M^{2}}{N^{2}} .
$$

Here $N^{2}=\partial b / \partial z$ is a measure of vertical stratification, $M^{2}=|\partial b / \partial y|$ is the analogous measure of horizontal stratification, and $b=-g \sigma / \rho_{o}$ is the buoyancy where $\sigma$ denotes the potential density.

If the transfer occurs in surfaces that have a slope that is one-half that of the mean isopycnal slope-as is the case in the growing phase of a linear Eady modelthen since $w^{\prime}=\left(\frac{1}{2}\right) s_{\text {rho }} v^{\prime}$,

$$
k_{v z}=k_{w y}=\frac{1}{2} s_{\text {rho }} k_{v y}
$$

and

$$
k_{w z}=\frac{1}{2} s_{\text {rho }} k_{v z}=\frac{1}{4} s_{\text {rho }}^{2} k_{v y} .
$$

In the limit of large Richardson number ( $\mathrm{Ri}$ is typically $10^{4}$ in the middle of the subtropical gyres dropping to $\sim 10^{2}$ in the regions of intense jets such as the Gulf Stream) GS argue that the horizontal transfer coefficient is given by

$$
k_{v y}=\alpha \frac{M^{2}}{N} l^{2}=\alpha \frac{f}{\sqrt{\mathrm{Ri}}} l^{2},
$$

where $f$ is the Coriolis parameter and the Richardson number of the large-scale flow (in thermal wind balance) is given by

$$
\operatorname{Ri}=\frac{N^{2}}{\left(\frac{\partial v}{\partial z}\right)^{2}}=\frac{N^{2} f^{2}}{M^{4}}
$$

Here $l$ is a measure of the meridional distance over which parcels of fluid are transferred in the baroclinic zone. Green (1970) argues that this distance is set by the width of the baroclinic zone. Stone (1972), however, suggests that the length scale of baroclinic eddies-the Rossby radius of deformation-is the appropriate eddy transfer scale. Finally, $\alpha$ is a constant of proportionality, which needs to be determined from eddy-resolving models or observations. By inspection of atmospheric data Green (1970) found that $\alpha=0.005$ for the poleward heat transfer of baroclinic eddies in the troposphere. Visbeck et al. (1996) found $\alpha=0.008 \pm 0.005$ in a (laboratory and numerical) study of convectively driven oceanic chimneys, remarkably close to that of Green.

Recognizing that $f / \sqrt{\mathrm{Ri}}$ is a measure of the growth rate of an Eady wave, Eq. (9) makes good physical sense setting $k_{v y}$ equal to an eddy velocity times a length scale. Thus, GS allows the $k$ to vary in space and time in a manner dependent on the large-scale density field as described by the large-scale Richardson number Ri and the transfer scale $l$, rather than setting them to a constant.

Broadly similar representations of poleward eddy heat fluxes have been used in atmospheric climate models by many workers (see Saltzman 1978, for a review) and are in use today (e.g., Stone and Yao 1990). More sophisticated variants of these ideas that focus on the trans- 
fer of potential vorticity flux were developed by White and Green (1984) for the atmosphere. They were applied to the ocean by Marshall (1981) in a study of a zonally averaged model of the Antarctic Circumpolar Current. There quasigeostrophic dynamics was assumed, which permits one to couch the closure problem in terms of quasigeostrophic potential vorticity and hence simultaneously capture the heat and vorticity transfer. The ideas of GS, however, have yet to be tried out in a threedimensional primitive equation ocean model.

\section{b. Transformed Eulerian mean: Gent and McWilliams}

In the middle atmosphere it was recognized that the distribution of chemical tracers cannot be accounted for simply by sources/sinks and the Eulerian mean circulation (with the mean, in the atmospheric context, being a zonal average) (see Andrews et al. 1987, chapter 9). Instead, the eddies transfer material essentially by a process similar to Stokes drift, and this transport is comparable in magnitude and often counterbalances the Eulerian mean meridional circulation. Andrews and McIntyre (1976) showed that one could add the waveinduced correction to the Eulerian circulation defined by the transformed Eulerian mean equations:

$$
\begin{aligned}
& \overline{\mathbf{v}}=\overline{\mathbf{v}}+\mathbf{v}^{*}=\overline{\mathbf{v}}+\frac{\partial \boldsymbol{\Psi}}{\partial z} \\
& \bar{W}=\bar{w}+w^{*}=\bar{w}-\boldsymbol{\nabla}_{h} \cdot \boldsymbol{\Psi},
\end{aligned}
$$

where $\boldsymbol{\Psi}$ is a (vector) streamfunction proportional to the buoyancy flux given by

$$
\boldsymbol{\Psi}=-\frac{\overline{\mathbf{v}^{\prime} b^{\prime}}}{N^{2}} .
$$

In (11) $\left(\mathbf{v}^{*}, w^{*}\right)$ are the "residual mean" velocities and denote the wave-induced tracer transport. Note that (11) and (12) apply the transformed Eulerian mean equations to three dimensions. This involves many assumptions about local growth and dissipation, which are discussed in length McDougall and McIntosh (1996) and Treguier et al. (1997). The above makes no attempt to parameterize the vertical eddy heat flux $\overline{w^{\prime} b^{\prime}}$.

These velocities are nondivergent and, for an equilibrated eddy field, are the appropriate advecting velocities for scalars; that is, Eq. (3) is replaced by

$$
\frac{\partial \bar{\tau}}{\partial t}+\overline{\mathbf{V}} \cdot \nabla_{h} \bar{\tau}+\bar{W} \frac{\partial \bar{\tau}}{\partial z}=\overline{S_{0}}-\bar{R}
$$

Here $R$ denotes an along-isopycnal mixing process, which can be represented in level models by a rotated diffusion tensor (Solomon 1971; Redi 1982). However, in the special case where $\tau$ is the buoyancy then the along-isopycnal mixing term $R$ is identically zero.

Because the transport velocities satisfy the nondivergence condition and should the rhs of (13) vanish (as it does for $\tau=b$ and $S_{0}=0$ ), then density surfaces are material surfaces and the large-scale flow can evolve in an adiabatic manner and the volume of fluid between isopycnal surfaces is conserved.

Gent and McWilliams (1990) argue that eddy transfer in the ocean is just such an adiabatic process and advocate that (13) be employed. If the eddy buoyancy flux is represented as horizontal transfer down the mean buoyancy gradient in analogy to (4)

$$
\overline{\mathbf{v}^{\prime} b^{\prime}}=-k^{\text {hor }} \nabla_{h} \bar{b},
$$

where $k^{\text {hor }}$ is a transfer coefficient whose variation has to be prescribed, then the residual mean velocities used by Gent and McWilliams (1990) are obtained:

$$
\begin{aligned}
& \mathbf{v}^{*}=\frac{\partial}{\partial z}\left(k \mathbf{s}_{\mathrm{rho}}\right) \\
& w^{*}=-\nabla_{h} \cdot\left(k \mathbf{s}_{\mathrm{rho}}\right),
\end{aligned}
$$

where $\mathbf{s}_{\text {rho }}$, the slope vector of the isopycnals, is given by (7).

Gent and McWilliams take $k$ in (14) to be a constant in the interior but set $k$ to zero on all boundaries:

$$
\left(\mathbf{v}^{*}+w^{*} \mathbf{k}\right) \cdot \mathbf{n}=0
$$

where $\mathbf{k}$ is a unit vertical vector and $\mathbf{n}$ normal to the boundary. This ensures that the eddy transfer process only redistributes heat within the fluid. As noted by Plumb and Mahlman (1987), the "effective transport velocities" $(\mathbf{V}, W)$ are not exactly equivalent to the Lagrangian mean when the diffusivities $k$ are spatially inhomogeneous (see Gent et al. 1995 and Andrews et al. 1987, for a discussion).

\section{c. A tutorial example}

Let us consider a baroclinic zone in which the initial density field is given by a $2 \times 2$ grid in the $y-z$ plane:

$$
\rho_{\text {initial }}=\left[\begin{array}{ll}
1 & 3 \\
1 & 3
\end{array}\right] .
$$

We are interested in the final properties of this system after rearrangement by baroclinic eddies to a state of minimal available potential energy and in the absence of external forcing.

If the eddy transfer is parameterized by horizontal diffusion, as in many large-scale ocean models, then, in the absence of forcing processes required to maintain it, the baroclinic zone will be diffused away. For example, one scenario would be

$$
\rho_{\mathrm{HD}, \text { final }}=\left[\begin{array}{ll}
2 & 2 \\
2 & 2
\end{array}\right],
$$

where strong diabatic mixing has occurred.

Next consider a rearrangement that conserves the volume of fluid within each density interval, as in the GM scheme. Since density surfaces are material surfaces, they will be rotated clockwise by the "effective transfer velocities" until the slope of the isopycnals is zero: 


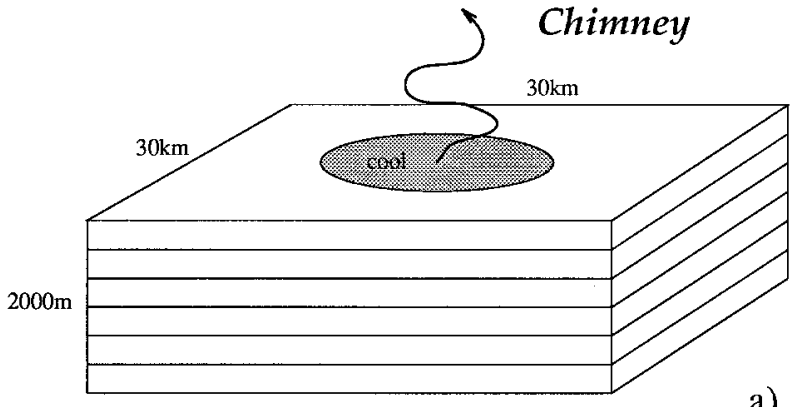

a)

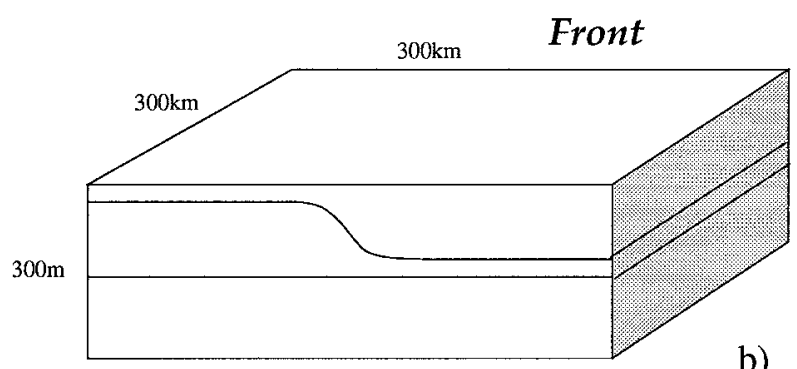

b)

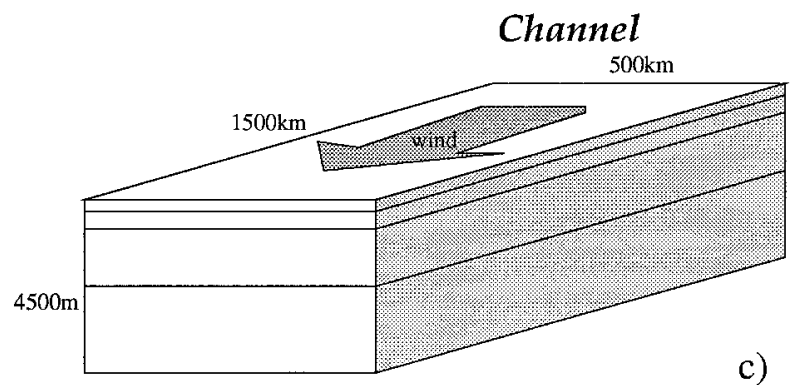

FIG. 1. Sketch of the three scenarios studied: (a) localized convection, (b) spindown of shallow front, and (c) a wind-driven channel.

$$
\rho_{\mathrm{GM}, \mathrm{final}}=\left[\begin{array}{ll}
1 & 1 \\
3 & 3
\end{array}\right] .
$$

Here there has been an adiabatic rearrangement without mixing across local density surfaces.

Finally, the GS scheme transfers properties along a surface with a slope one-half of that of the isopycnal surface at a rate that is a function of the slope. The Green and Stone scheme will yield a final density field somewhat between the other two limiting cases. For example,

$$
\rho_{\mathrm{GS}, \text { final }} \sim\left[\begin{array}{ll}
1.5 & 1.5 \\
2.5 & 2.5
\end{array}\right] .
$$

We see that the final density fields are quite different but in all cases the end state is characterized by an absence of available potential energy. The potential energy released in the rearrangement is greatest in GM, zero in the case of horizontal diffusion, and intermediate in the case of GS.
We conclude from this thought experiment that in those places in the ocean where geostrophic eddy dynamics is essentially adiabatic, the approach of GM is to be preferred. However, major baroclinic eddy activity occurs in strong frontal regions of the ocean (such as the Gulf Stream and the ACC), places that are also subject to vigorous air-sea fluxes and diabatic processes. But GS implicitly makes rather strong assumptions about the nature of the mixing process on the small scale: that the mixing is essentially "slaved" to (its rates controlled by) the geostrophic eddy scale. It is not clear to what extent this is appropriate in the ocean. Finally, it should be noted that the GM scheme can allow for explicit diapycnal mixing if a more general form of the rotated mixing tensor $R$ is used.

\section{Implementation of tracer parameterization schemes}

It is possible to succinctly represent GS and GM in one mathematical framework, if we make use of a tensor $\mathbf{K}$, such that the subgrid tracer transport of Eq. (3) is given in terms of mean quantities; thus,

$$
\left[\begin{array}{l}
\overline{v^{\prime} \tau^{\prime}} \\
\overline{w^{\prime} \tau^{\prime}}
\end{array}\right]=-\mathbf{K}_{2 \mathrm{D}}\left(\nabla_{2 \mathrm{D}} \bar{\tau}\right) .
$$

In the following we will discuss how this tensor formalism is implemented in a two-dimensional version of the tracer equation $(3, \mathrm{~A} 5)$.

\section{a. Eddy transfer parameterized by horizontal diffusion (HD)}

We can implement transfer of the form (4) and (5) by setting the off-diagonal tensor elements to zero:

$$
\mathbf{K}=k_{v y}\left[\begin{array}{ll}
1 & 0 \\
0 & \epsilon
\end{array}\right],
$$

where $k_{v y}$ is the horizontal transfer coefficient and $\epsilon=$ $k_{w z} / k_{v y} \ll 1$ denotes the ratio between horizontal and vertical transfer.

\section{b. The GS parameterization}

In order to implement the transfer coefficient formalism of GS we can employ a rotated diffusion tensor similar to the one given by (18) and transfer tracers along surfaces of slope $s$. In the desired direction of transfer, that is, along this sloping surface, the $\mathbf{K}$ tensor is of the form

$$
\mathbf{K}_{s}=k_{s}\left[\begin{array}{cc}
1 & 0 \\
0 & \epsilon_{s}
\end{array}\right],
$$

where $\epsilon_{s}$ denotes the ratio between off axis (normal to $s$ ) and along axis (along $s$ ) transfer. Rotation to Cartesian coordinates yields 
TABLE 1. Eddy-resolving model parameter.

\begin{tabular}{|c|c|c|c|c|}
\hline Parameter & Units & Chimney & Front & Wind \\
\hline $\begin{array}{l}\text { Model } \\
\text { Vertical coordinate } \\
\text { Hydrostatic } \\
\text { Domain }\end{array}$ & & $\begin{array}{l}\text { MIT } \\
\text { Pressure } \\
\text { No } \\
\text { Double per. }\end{array}$ & $\begin{array}{l}\text { MICOM } \\
\text { Density } \\
\text { Yes } \\
\text { Channel }\end{array}$ & $\begin{array}{l}\text { MIT } \\
\text { Pressure } \\
\text { Yes } \\
\text { Channel }\end{array}$ \\
\hline $\begin{array}{l}\text { Horizontal domain size } \\
\text { Domain depth } \\
\text { Horizontal grid size } \\
\text { Vertical grid size } \\
\text { Number of vertical levels/layers }\end{array}$ & $\begin{array}{l}\mathrm{km} \\
\mathrm{m} \\
\mathrm{km} \\
\mathrm{m}\end{array}$ & $\begin{array}{l}30 \times 30 \\
2000 \\
0.25 \\
100 \\
20\end{array}$ & $\begin{array}{l}300 \times 300 \\
300 \\
2 \\
-\quad 3\end{array}$ & $\begin{array}{l}500 \times 1500 \\
4500 \\
10 \\
25-400 \\
20\end{array}$ \\
\hline $\begin{array}{l}\text { Stratification (N/f) } \\
\text { External Rossby radius (NH/f) } \\
\text { Burger number (NH/fL) }\end{array}$ & $\mathrm{km}$ & $\begin{array}{l}5 \\
4 \\
0.26\end{array}$ & $\begin{array}{l}54 \\
17 \\
0.06\end{array}$ & $\begin{array}{l}15 \\
66 \\
0.13\end{array}$ \\
\hline $\begin{array}{l}\text { Horizontal/isopycnal diffusivity } \\
\text { Biharmonic diffusivity } \\
\text { Vertical/diapycnal diffusivity } \\
\text { Vertical viscosity } \\
\text { Bottom friction }\end{array}$ & $\begin{array}{l}\mathrm{m}^{2} \mathrm{~s}^{-1} \\
\mathrm{~m}^{4} \mathrm{~s}^{-1} \\
\mathrm{~m}^{2} \mathrm{~s}^{-1} \\
\mathrm{~m}^{2} \mathrm{~s}^{-1} \\
\mathrm{~s}^{-1}\end{array}$ & $\begin{array}{r}10 \\
-\quad 0.1 \\
-\quad 0.1\end{array}$ & $\begin{array}{r}5 \\
- \\
0 \\
-\end{array}$ & $\begin{array}{l}- \\
10^{10} \\
10^{-5} \\
10^{-3} \\
10^{-4}\end{array}$ \\
\hline $\begin{array}{l}\text { Rotation rate } f \\
\text { Length of integration }\end{array}$ & $\begin{array}{l}\mathrm{s}^{-1} \\
\text { days }\end{array}$ & $\begin{array}{l}10^{-4} \\
10\end{array}$ & $\begin{array}{c}10^{-4} \\
100\end{array}$ & $\begin{array}{l}10^{-4} \\
3500\end{array}$ \\
\hline $\begin{array}{l}\text { Surface heat loss } Q \\
\text { Surface wind stress }\end{array}$ & $\begin{array}{l}\mathrm{W} \mathrm{m}^{-2} \\
\mathrm{~Pa}\end{array}$ & 800 & - & $-_{0.2}$ \\
\hline
\end{tabular}

$$
\mathbf{K}=\frac{k_{s}}{\left(s^{2}+1\right)}\left[\begin{array}{cc}
1+s^{2} \boldsymbol{\epsilon}_{s} & s\left(1-\boldsymbol{\epsilon}_{s}\right) \\
s\left(1-\boldsymbol{\epsilon}_{s}\right) & s^{2}+\boldsymbol{\epsilon}_{s}
\end{array}\right],
$$

where $s$ is the slope of the surface of exchange. We arrive at Green's transfer coefficients (8) from (20) by setting the rotation angle $s=(1 / 2) s_{\text {rho }}$ to be one-half the isopycnal slope and $\epsilon_{s}=0$. Note that this is strictly true only for small slopes $\left(s^{2}<1\right)$. One can readily allow the transfer coefficients $k_{s}$ to vary in space according to (9). Details of how this might be done are described subsequently.

\section{c. GM parameterization}

The GM scheme can be implemented by splitting the diffusion tensor $\mathbf{K}$ into two parts (Plumb and Mahlman 1987):

$$
\mathbf{K}=\mathbf{K}_{\mathrm{adv}}+\mathbf{K}_{\mathrm{iso}},
$$

where $\mathbf{K}_{\mathrm{adv}}$ is an antisymmetric tensor representing the effective transport velocities and $\mathbf{K}_{\text {iso }}$ is a symmetric tensor that denotes mixing along isopycnals (20) with $s=s_{\text {rho }}$ given by the isopycnal slope and $\epsilon_{s}=0$.

The antisymmetric tensor is given by

$$
\mathbf{K}_{\mathrm{adv}}=k_{s}\left[\begin{array}{cc}
0 & s_{\mathrm{rho}} \\
-s_{\text {rho }} & 0
\end{array}\right] .
$$

The flux divergence $\boldsymbol{\nabla}_{h} \cdot\left(\mathbf{K}_{\text {adv }} \nabla \tau\right)$ given by the residual mean velocities $\left(\mathbf{v}^{*}, w^{*}\right)(15)$ (see also Andrews et al. 1987, chapter 9).

\section{d. The hybrid eddy transfer scheme (NEW)}

Gent and McWilliams (1990) choose the value of the diffusivity $k_{s}$ from Eq. (21) independent of the largescale density field and assumed $k_{s}$ to be constant in time and space (McWilliams and Gent 1994; Danabasoglu et al. 1994; Gent et al. 1995). Here we will replace $k^{\text {hor }}$ of (14) by the horizontal eddy transfer coefficient employed by the GS scheme (9).

To prescribe the form of the transfer coefficients $k_{v y}$ [Eq. (19)], we adopt the following procedure: First, evaluate a local growth rate from the isopycnal slope $\left(s_{\text {rno }}\right)$ and the stratification $(N)$ averaged over the vertical column:

$$
\frac{1}{t_{e}}=\frac{f}{\sqrt{\mathrm{Ri}}} \sim \frac{1}{H} \int \frac{M^{2}}{N} d z=\frac{1}{H} \int s_{\mathrm{rho}} N d z .
$$

Second, the spatial form of the growth rate field is used to obtain a characteristic length scale $l$. The minimum length scale is chosen to be the Rossby radius $(N H / f)$ or the grid scale, whichever is greater. In regions of large growth rate $l$ is gradually increased to the width of the baroclinic zone. This is in accord with the argument of Green (1970) and supported by the homogeneous model of fully developed baroclinic instability explored by Larichev and Held (1995), who assume equipartition of eddy kinetic and potential energy and equate it to the available potential energy of the mean flow at the scale where the inverse energy cascade is halted-here the full extent of the baroclinic zone.

The width of the baroclinic zone is defined as the width of the region where the local growth rate exceeds $10 \%$ of the maximum growth rate of the field. Multi- 


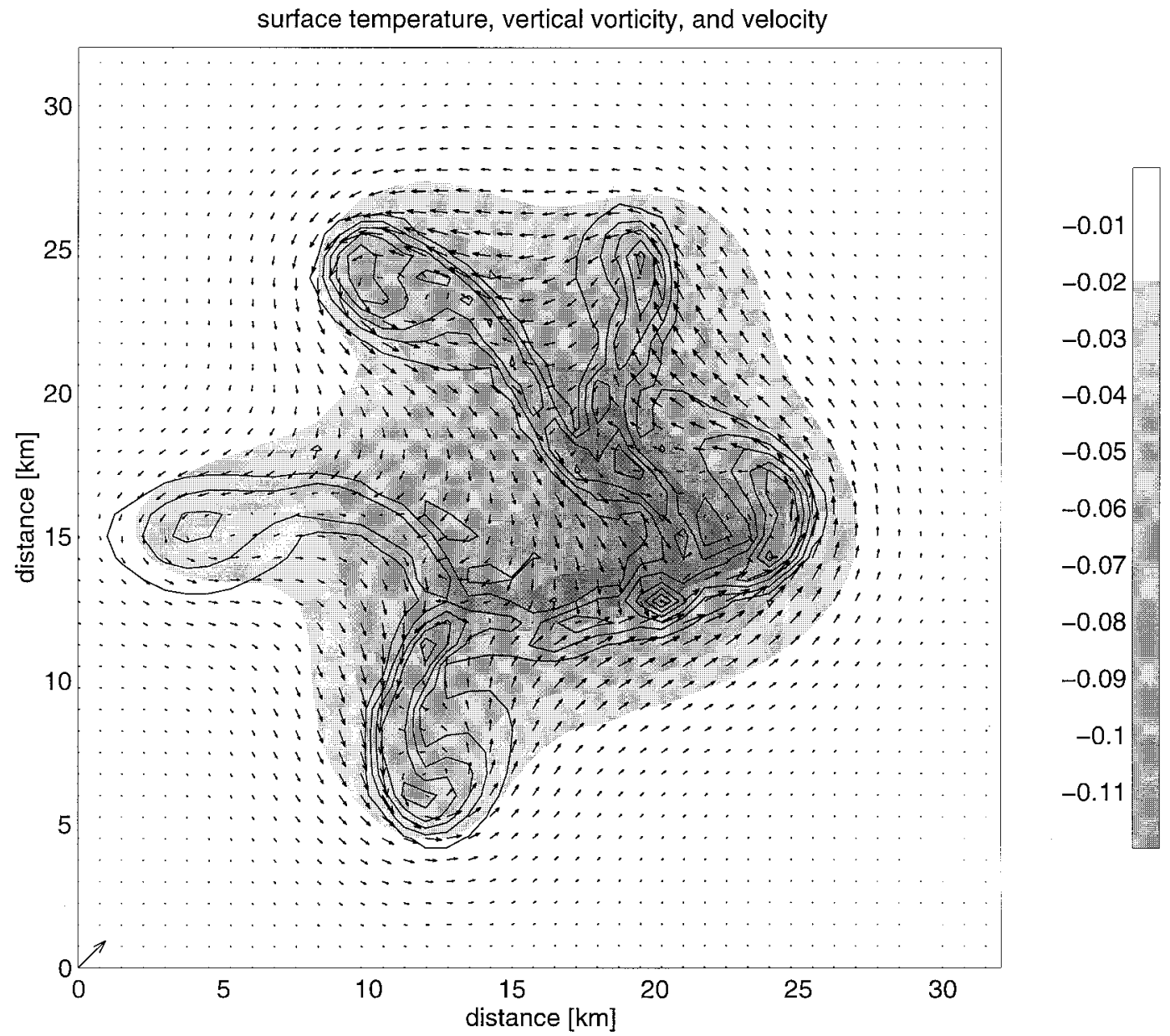

FIG. 2. Surface currents, vertical component of vorticity, and temperature at day 6. Every third vector is displayed and the maximum velocity is $0.33 \mathrm{~cm} \mathrm{~s}^{-1}$. The temperature is shaded using a $0.02-\mathrm{K}$ interval corresponding to a density interval of $0.004 \mathrm{~kg} \mathrm{~m}^{-3}$, and contours of relative vorticity are included (solid lines)

plying the growth rate $\left(1 / t_{e}\right)$ by this mixing length scale $\left(l^{2}\right)$ and a constant of proportionality $(\alpha)$ we obtain the transfer coefficient $k_{v y}(9)$. In (9) $\alpha$ is the primary "tunable" parameter and will be varied systematically to determine its optimum value.

\section{Numerical experiments using a two-dimensional model}

Our strategy is to set up three-dimensional model calculations that resolve the baroclinic eddy field in interesting dynamical regimes. We then average these threedimensional fields zonally or azimuthally and compare them to a parameterized two-dimensional model in the $y-z$ plane. The two-dimensional model, described in the appendix, assumes a balanced flow at small Rossby number and accommodates the four eddy flux parameterization schemes outlined in sections 2 and 3 .

- HD denotes experiments in which eddies are parameterized as horizontal diffusers of heat and salt as- suming a constant diffusion coefficient $k$ using Eqs. (3), (4).

- GS denotes the Green-Stone scheme in which the transfer coefficients depend on the density field according to (9), and heat and salt are transferred along surfaces that have a slope one-half that of the isopycnal slope using Eqs. (3), (6), (8), and (9).

- GM denotes the Gent and McWilliams scheme and employs the residual mean velocities and isopycnal mixing with constant transfer coefficients using Eqs. (13), (11), (12), and (14).

- NEW denotes a hybrid scheme that combines the transformed Eulerian mean formalism (GM) with spatially and temporally varying transfer coefficients computed as in (GS) using Eqs. (13), (11), (12), (14), and (9).

In some cases we have carried out additional 2D model simulations that do not include eddy flux parameterizations; one is MIX in which only convective adjust- 

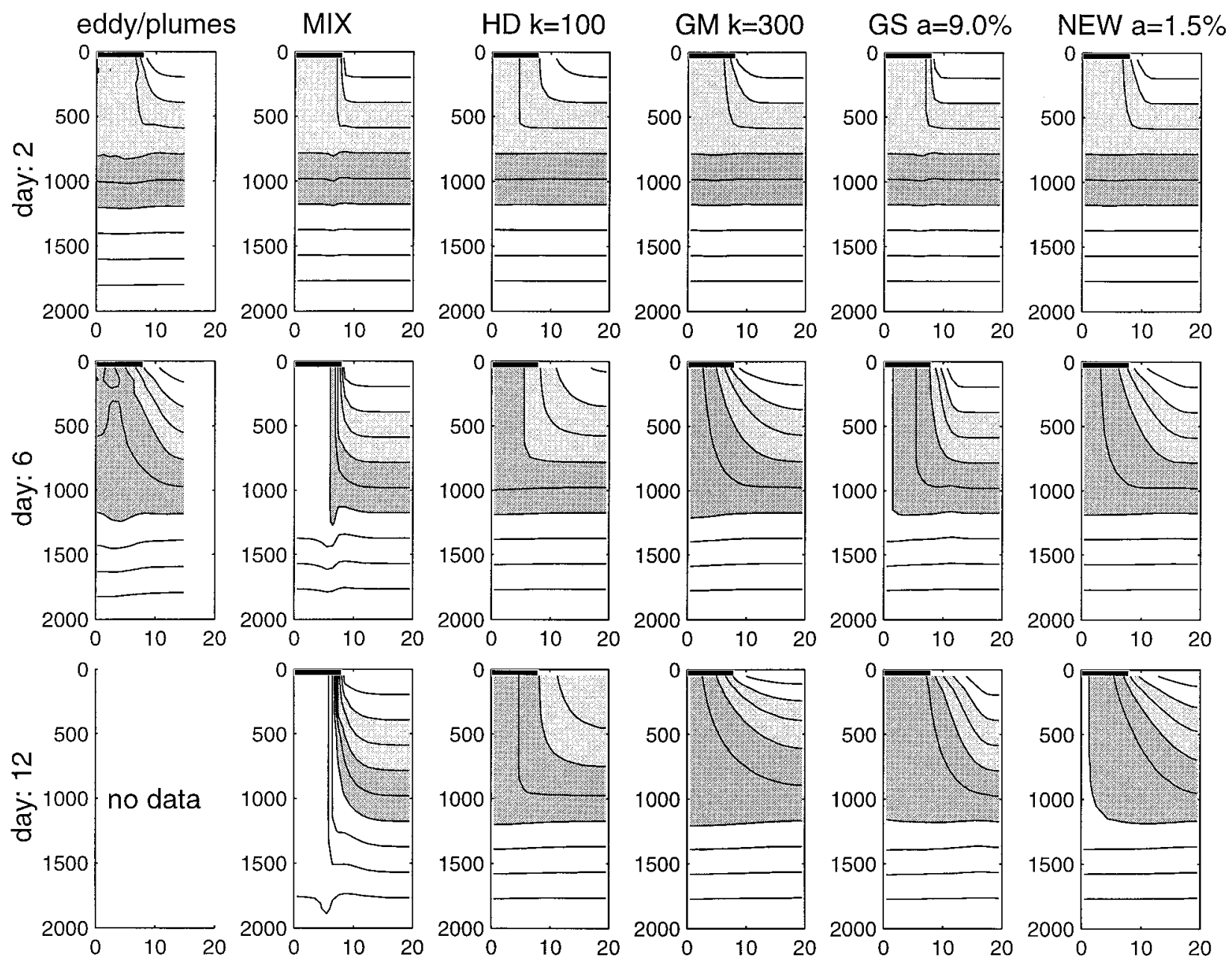

FIG. 3. Radial averaged density as a function of depth (in $\mathrm{m}$ ) and radius (in $\mathrm{km}$ ) for day 2, 6, and 12 for the nonhydrostatic eddy-resolving model (left column) and the parameterized 2D model for different parameterization schemes (see text for details). The contour interval is $0.005 \mathrm{~kg} \mathrm{~m}^{-3}$ and two isopycnal layers are shaded. The black bar at the surface indicates to cooling region.

ment is included and the other is ISO where only mixing along isopycnal surfaces is included.

All of the parameterization schemes have one free parameter that controls the efficiency of the release of available potential energy. In the case HD and GM it is the transfer coefficient $k$, while for the GS and NEW schemes it is the coefficient $\alpha$ of (9).

We have studied three scenarios (Fig. 1): 1) a convective chimney in which an initially uniformly stratified fluid of 2-km depth is cooled over a disk of $16-\mathrm{km}$ diameter; 2) a shallow upper-ocean front in a periodic channel of width $300 \mathrm{~km}$ and depth $300 \mathrm{~m}$, and 3) an exponentially stratified ocean in a channel $1500 \mathrm{~km}$ long and $500 \mathrm{~km}$ wide, forced at its upper surface by wind and retarded by friction at its bottom at a depth of 4.5 $\mathrm{km}$. In all eddy-resolving simulations, the Coriolis parameter was constant throughout the domain ( $f$ plane). Subgrid-scale mixing was represented by either Laplacian or biharmonic diffusion. The explicit calculations were performed using two different ocean models: the
MICOM isopycnal model (Bleck and Boudra 1986) for the shallow front, a nonhydrostatic version of the MIT model (Marshall et al. 1996a,b) for the convective chimney, and a hydrostatic version of the same model for the wind-driven channel. The eddy-resolving model parameters are summarized in Table 1.

We evaluate the skill of the parameterization schemes by comparing resolved and parameterized models using three different criteria.

- Available potential energy: We define the available potential energy as the energy that could be obtained by a process that adiabatically redistributes the fluid parcels to a statically stable state of rest. A qualitative measure of the available potential energy is obtained by computing the ratio of the mean density over onehalf the domain (to the north of an unstable front, for example) to the mean density over the whole domain. A field of zero available potential energy-all isopycnals are horizontal-has a ratio of 1 ; smaller or 

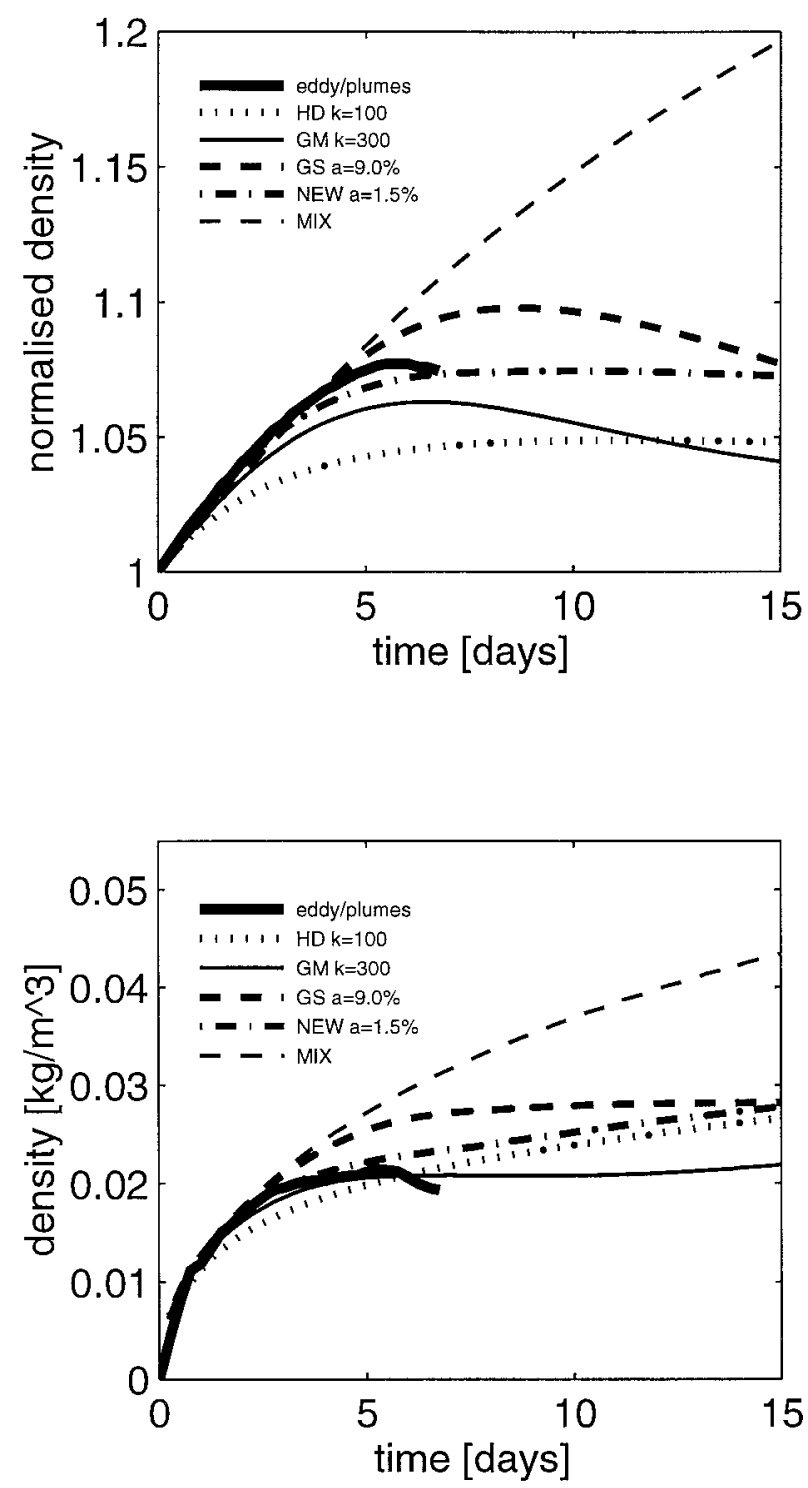

FIG. 4. (a) Evolution of the mean density under the cooling region normalized by the total mean density. (b) Evolution of the mean surface density under the cooling region.

larger values indicate that potential energy is available for rearrangement. We will use this measure to "optimize" the choice of $\alpha$ or $k$ for each of the different schemes.

- Diapycnal mixing: A measure of the diapycnal mixing is obtained by comparing the potential energy of the evolving fields. In an unforced system, adiabatic rearrangement of fluid parcels will result in the smallest absolute value of potential energy. Vertical mixing, however, tends to increase the potential energy, while horizontal diffusion leaves it unchanged (see the tutorial example of section 3).

- Explained variance: A measure of the spatial similarity of chosen fields is provided by the explained spatial variance, which is defined here by
TABLE 2. Two-dimensional model parameters.

\begin{tabular}{lllll}
\hline \hline \multicolumn{1}{c}{ Parameter } & Units & Chimney & Front & Wind \\
\hline Geometry & & Cylinder & Channel & Channel \\
Vertical resolution & $\mathrm{m}$ & 100 & 16 & 30 \\
Horizontal resolution & $\mathrm{km}$ & 1 & 15 & 25 \\
Relaxation time & days & 2 & 5 & 10 \\
Newtonian damping $r_{i}$ & $\mathrm{~s}^{-1}$ & $10^{-6}$ & $10^{-6}$ & $10^{-6}$ \\
Bottom friction $r_{b}$ & $\mathrm{~s}^{-1}$ & - & - & $10^{-4}$ \\
\hline
\end{tabular}

$$
C=\frac{\sum\left(\tau_{3 D}(y, z)-\bar{\tau}_{3 D}(z)\right)^{2}-\Sigma\left(\tau_{2 D}(y, z)-\tau_{3 D}(y, z)\right)^{2}}{\sum\left(\tau_{3 D}(y, z)-\bar{\tau}_{3 D}(z)\right)^{2}},
$$

where $\tau_{3 D}$ is the zonally or (azimuthally) averaged density field in the resolved model, $\tau_{2 D}$ the $2 \mathrm{D}$ model density field, and the overbar denotes a spatial mean. We have chosen this definition of the variance, in which the mean stratification of the reference has been removed, to highlight horizontal variations. The explained variance (23) at a chosen time allows one to obtain a sensitive measure of the fidelity of the

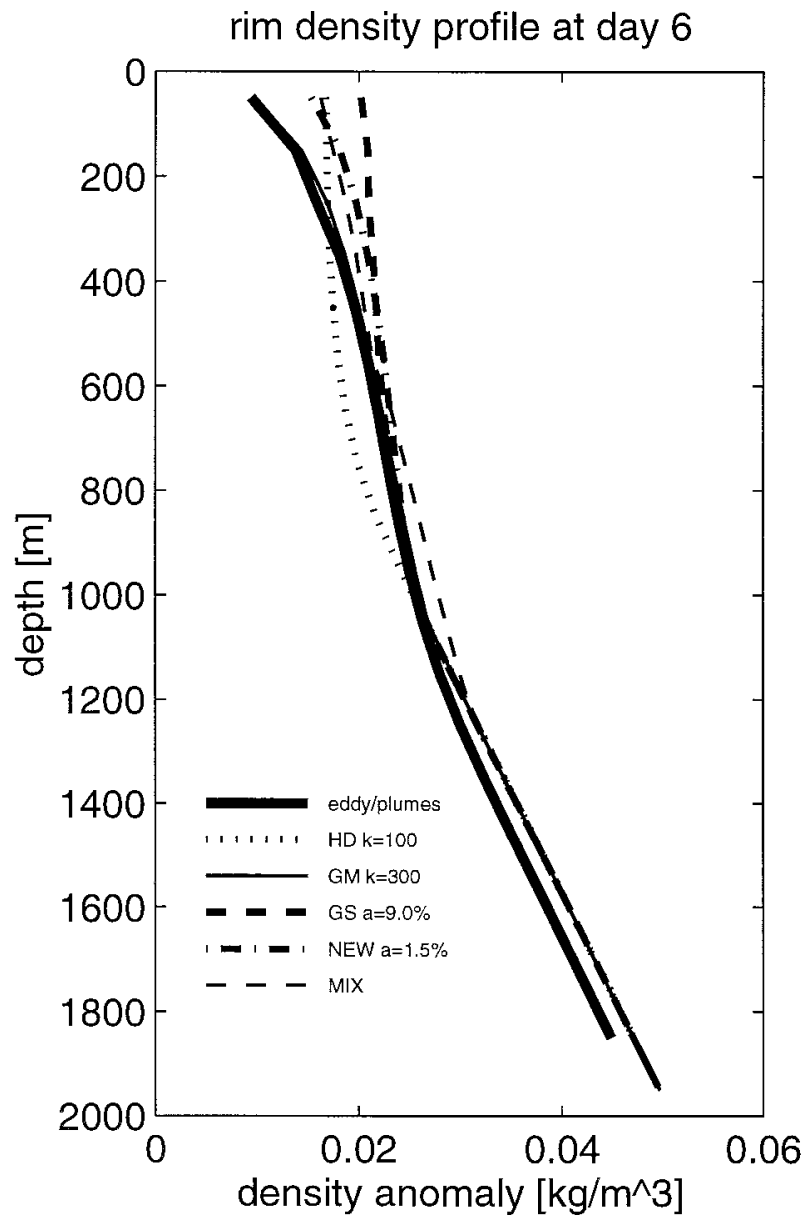

FIG. 5. Density profiles at the edge of the cooling region for different parameterization and the eddy-resolving model. 


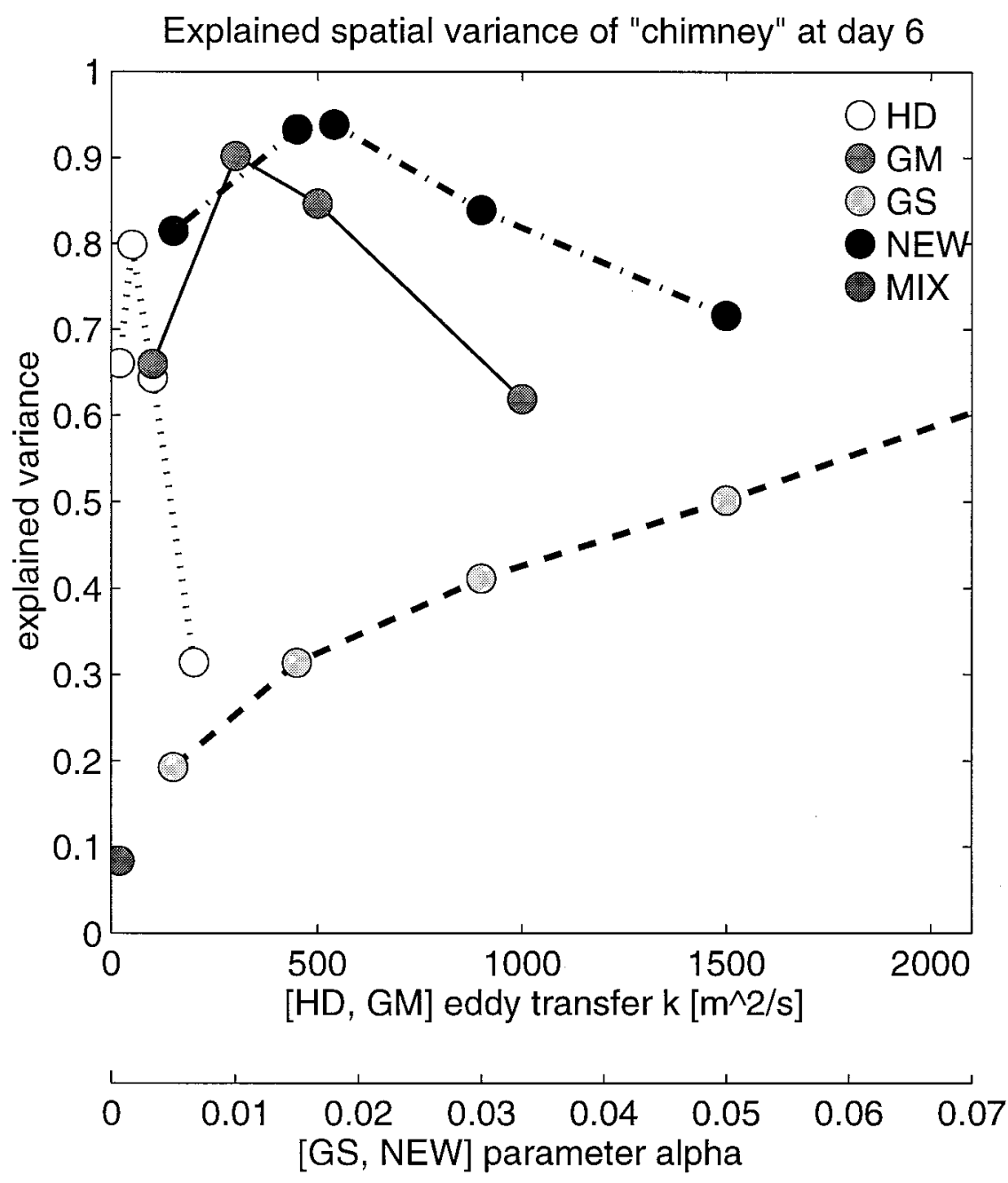

FIG. 6. Explained spatial variance referenced to the eddy-resolving model field at day 6 for the different parameterization versus $k$ for HD and GM and versus $\alpha$ for GS and NEW.

parameterization schemes and so can be used for tuning purposes.

a. A convective chimney driven by buoyancy forcing

The first scenario studied is appropriate to water mass transformation by open ocean convection at high latitudes. The reference experiment is an idealized convective scenario using an eddy-resolving nonhydrostatic model with 250-m horizontal resolution (Visbeck et al. 1996). A linearly stratified volume of water $(N / f=5)$ is cooled at the surface at a rate of $800 \mathrm{~W} \mathrm{~m}^{-2}$ (corresponding to a buoyancy loss of $B_{0}=3.8 \times 10^{-7} \mathrm{~m}^{2}$ $\mathrm{s}^{-3}$ ) over a disk of $16-\mathrm{km}$ diameter and zero elsewhere. Under the cooling disk a cylinder of dense mixed layer fluid-a chimney-forms; the underlying dynamics are discussed in Jones and Marshal (1993) and Maxworthy and Narimousa (1994). Visbeck et al. (1996), however, considered the limiting case in which the depth to which the chimney penetrates is arrested by lateral heat transfer due to baroclinic eddies that sweep stratified water into the chimney at the surface and move cold convected

TABLE 3. Coefficients for the eddy flux parameterizations.

\begin{tabular}{lccccc}
\hline \multicolumn{1}{c}{ Scheme } & Variable & Units & Chimney & Front & Wind \\
\hline Horizontal diffusion HD & $k_{h}$ & $\mathrm{~m}^{2} \mathrm{~s}^{-1}$ & 50 & 500 & 3000 \\
Half isopycnal slope diffusion GS & $\alpha$ & & 0.09 & 0.025 & 0.015 \\
Transformed Eulerian mean GM & $k_{g}$ & $\mathrm{~m}^{2} \mathrm{~s}^{-1}$ & 300 & 500 & 2000 \\
Hybrid scheme (GM and GS) NEW & $\alpha$ & & 0.015 & 0.015 & 0.010 \\
\hline
\end{tabular}



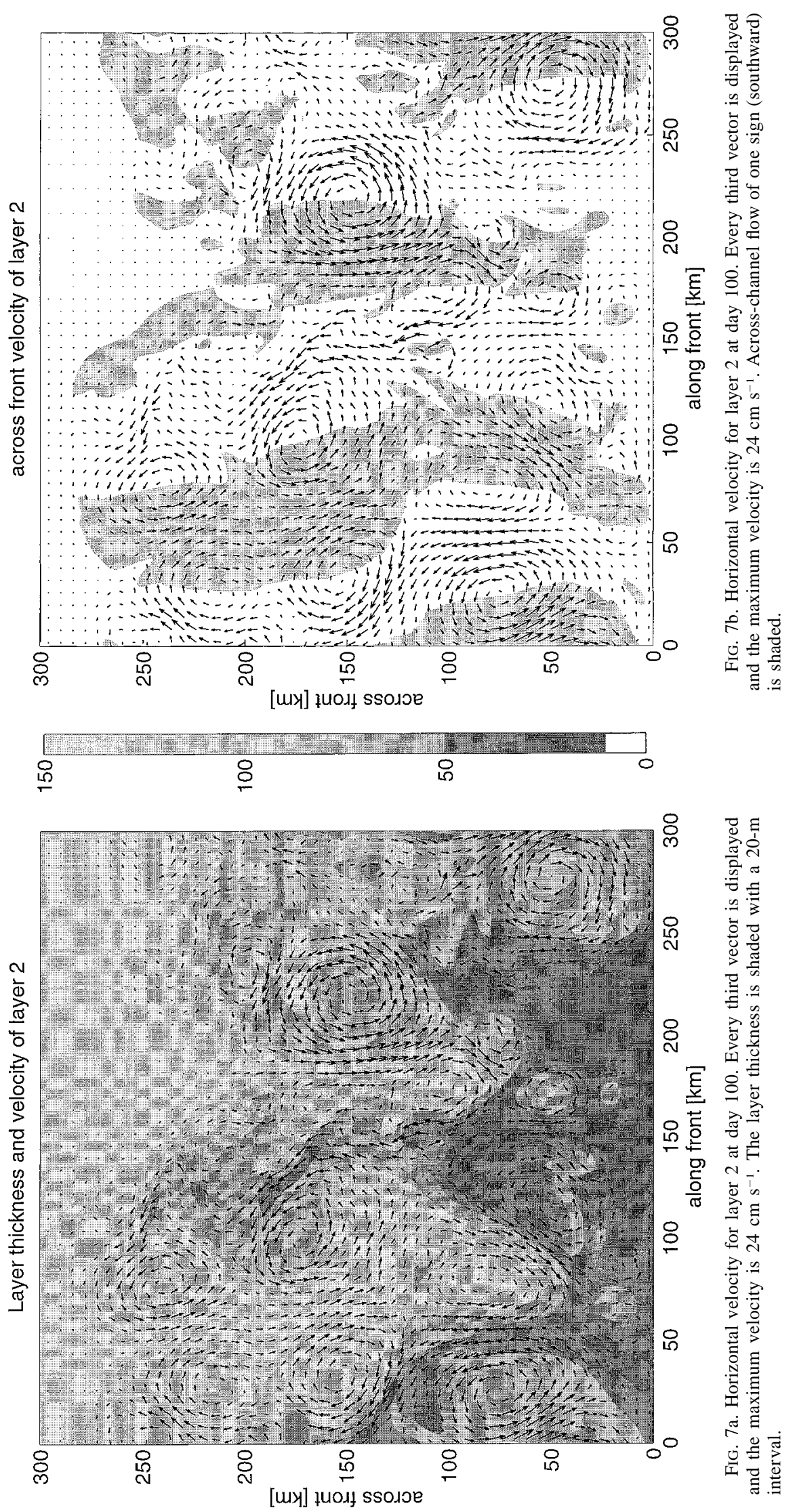

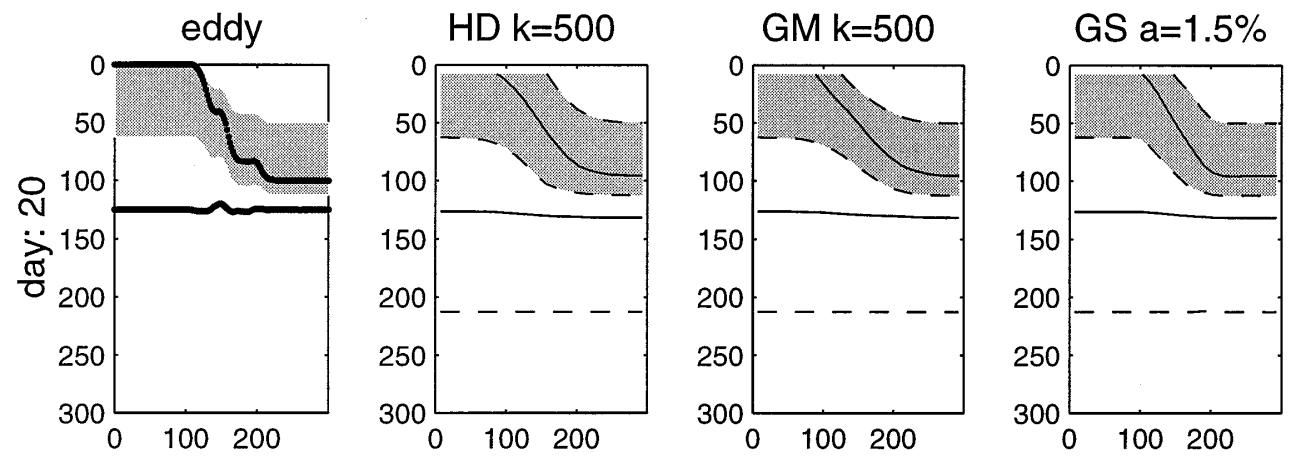

NEW $a=1.5 \%$
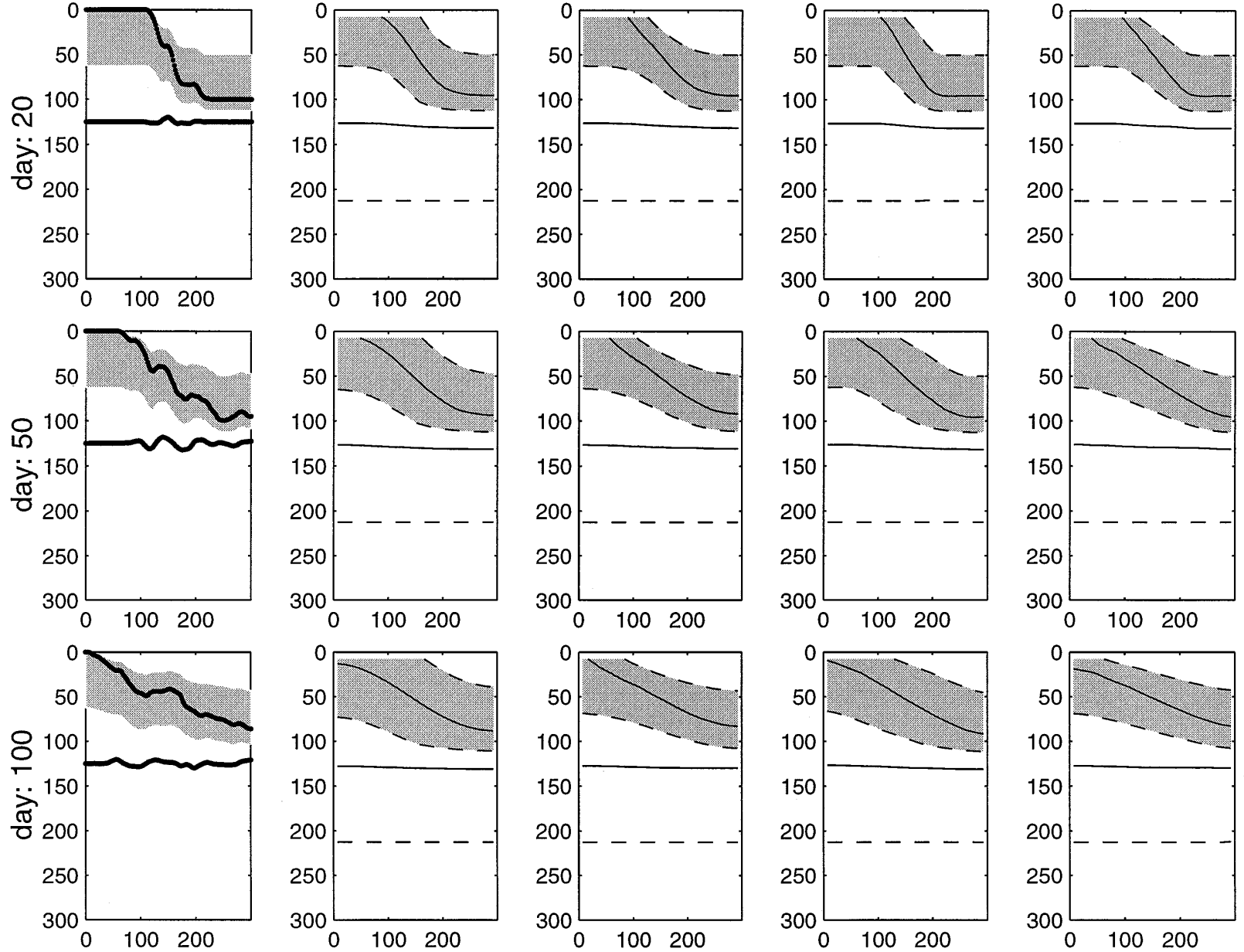

FIG. 8. Evolution of the alongchannel averaged layer depth for day 20, 50, and 100 for the isopycnal eddy-resolving model (left column) and the parameterized 2D model for different parameterization schemes (see text for details). Thick lines represent the depth of layer interfaces of the resolving model, while thin lines denote the corresponding density contours in the 2D model field. The density layer between 0.15 and $0.45 \mathrm{~kg} \mathrm{~m}^{-3}$ is shaded.

water outward below. A snapshot of the surface temperature and velocity field at day 6 from such an experiment shows the breakup of the chimney due to a mode-five instability of the rim current (Fig. 2). Azimuthally averaged sections across the baroclinic zone show the generation of a chimney due to convective mixing and its breakup by lateral fluxes due to baroclinic eddies (Fig. 3, left column).

The depth to which convection penetrates in the chimney is reflected in the evolution of the surface density under the cooling region (Fig. 4b). To the extent that the convection is one-dimensional and entrainment from below can be neglected, the penetration of a mixed layer into a stratified fluid subject to constant buoyancy loss at its surface is given by

$$
h=\frac{\left(2 B_{0} t\right)^{1 / 2}}{N}, \quad b_{\text {mix }}^{\prime}=N^{2} h=\left(2 B_{0} t\right)^{1 / 2} N .
$$

The buoyancy anomaly $\left(b_{\text {mix }}^{\prime}\right)$ thus grows with the square root of time in the absence of lateral buoyancy fluxes (Turner 1973; Visbeck et al. 1996). From day 5 onward, however, the lateral buoyancy transfer becomes sufficiently large that it ultimately offsets the surface buoyancy loss and a quasi-steady state is established. The maximum depth to which convection penetrates is given by (see Visbeck et al. 1996):

$$
h_{\text {final }}=\gamma \frac{\left(B_{0} r\right)^{1 / 3}}{N},
$$

where $r$ is the chimney radius and $\gamma$ is a proportionality constant related to the $\alpha$ that appears in (2) by $\gamma=$ $(1 / 2 \alpha)^{1 / 3}$. Study of several laboratory and numerical experiments of convectively driven chimneys shows (see Visbeck et al. 1996; Whitehead et al. 1996) that $\gamma=$ $3.9 \pm 0.9$, implying that $\alpha=0.008 \pm 0.005$ close to the 0.005 obtained by Green (1970) in his studies of baroclinic eddies in the atmosphere. This is an interesting reference value against which to consider the $\alpha$ 

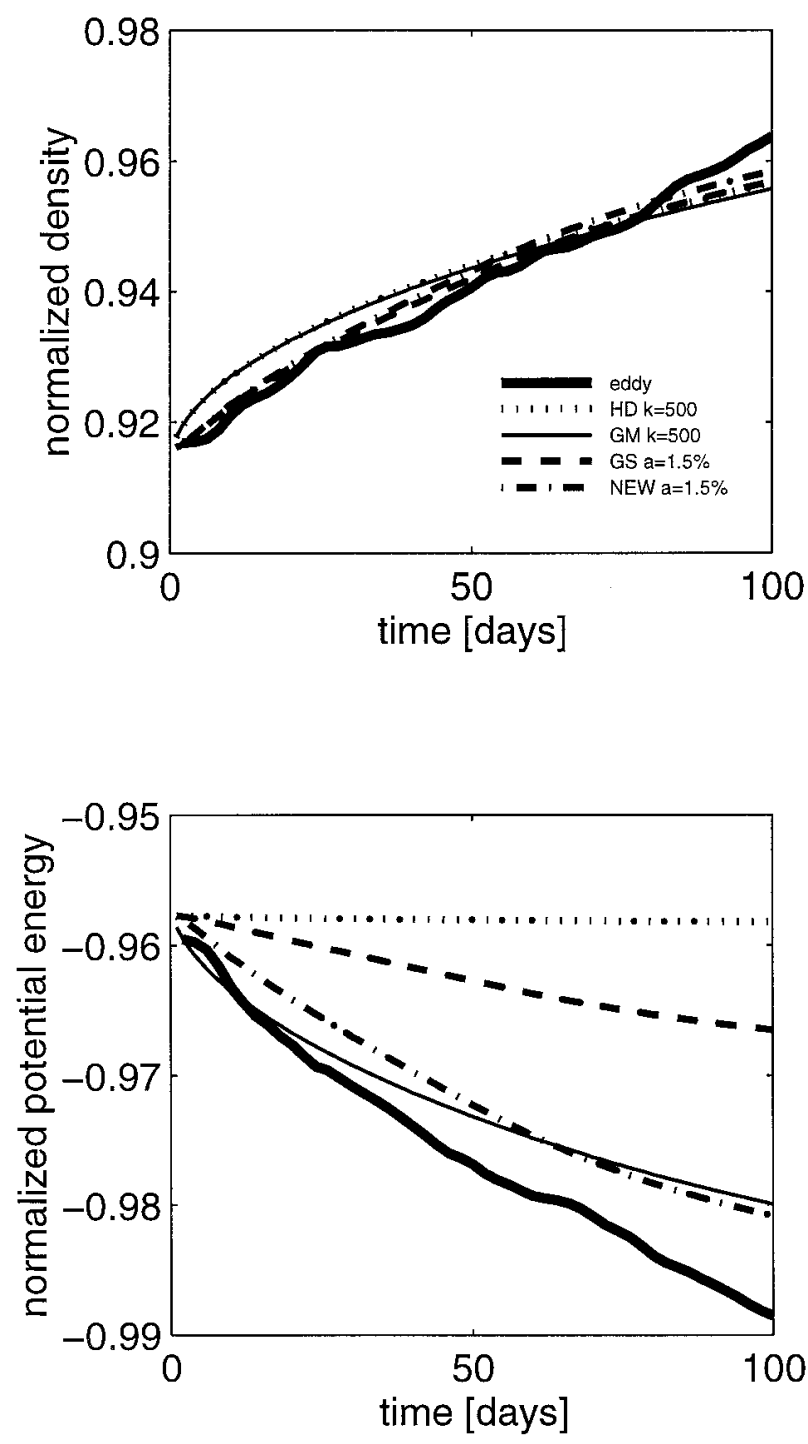

FIG. 9. Evolution of (a) the right half domain-averaged density divided by the total mean density and (b) the mean potential energy normalized by the minimum potential energy versus time for the eddyresolving isopycnal model and all parameterizations.

deduced from our parameterized model by comparison with the resolved models.

The 2D model is configured for an azimuthally averaged domain and convective mixing is represented by a convective adjustment scheme (Table 2). Figure 4 a shows that all eddy flux parameterizations are able to balance the surface buoyancy loss and establish some kind of quasi-steady state except the run MIX, which represents a case where the eddy transfer parameterizations were turned off and only frictional spindown and convective adjustment were included (Fig. 4b). However, the details of the chimneys vary considerably; the GM and NEW schemes seem most able to capture the form observed in the eddy-resolving model (Fig. 3). They predict a large lateral buoyancy flux near the sur- face associated with the strong baroclinic zone of the rim current. At depth the convected fluid is advected outward and appears as a layer of reduced stratification (Fig. 5).

The azimuthally averaged field from day 6 of the eddy-resolving model was used as a reference to explore the sensitivity of the parameterization schemes with respect to their tunable parameters (Fig. 6). The GM scheme shows a plateau of acceptable performance centered around a transfer coefficient of $300 \mathrm{~m}^{2} \mathrm{~s}^{-1}$ (Table $3)$. Horizontal diffusion shows a steep drop in performance, which indicates that a $k$ of $1000 \mathrm{~m}^{2} \mathrm{~s}^{-1}$ or more will yield poor results in these water mass formation regions. The optimum value of $\alpha=0.015$ for the NEW scheme, however, is within a factor of 2 of the $\alpha=$ $0.008 \pm 0.005$ deduced from laboratory and numerical experiments (Visbeck et al. 1996; Whitehead et al. 1996). The $k$ implied by (9) is $\sim 300 \mathrm{~m}^{2} \mathrm{~s}^{-1}$.

\section{b. Spindown of a baroclinic front}

The second scenario considered is a shallow upperocean front typical of those found in Ekman convergence zones of the subtropical North Atlantic and North Pacific. Spall (1995) simulated subduction and crossfrontal exchange for such a shallow upper-ocean front using an eddy-resolving isopycnal model (MICOM, Bleck and Boudra 1986). The model has three active isopycnal layers of $0.3 \mathrm{~kg} \mathrm{~m}^{-3}$ density difference in a periodic channel of $300 \mathrm{~km} \times 300 \mathrm{~km}$ width and $300-\mathrm{m}$ depth (Table 1) and was integrated for 100 days.

The front becomes baroclinically unstable and an energetic eddy field evolved (Fig. 7a). By day 100 the baroclinic zone spans the whole channel of several Rossby radii in width. Several eddies are found in the domain, but there appears to be some organization. Figure $7 \mathrm{~b}$ shows the same velocity field but the southward velocity is shaded. Long bands of across-frontal flow exist, which possibly transfer fluid a distance of several eddy scales from one side of the front to the other.

Averaging the three-dimensional fields along the front allows one to inspect the eddy transfer across the front in the mean. Here we have assumed that the spatial average over many eddies is equivalent to a temporal average over eddy life cycles. The evolution of the isopycnal layer thickness (Fig. 8, left column) shows that baroclinic eddies act to mix layer thickness (and potential vorticity) on isopycnal surfaces and thereby release the potential energy stored in the inclined density surfaces.

The corresponding 2D model experiments were initialized with a similar density field by interpolating between layer interfaces onto a Cartesian $y-z$ grid with 15 $\mathrm{km}$ horizontal and $16 \mathrm{~m}$ vertical resolution (Table 2). Again $\alpha$ and $k$ were varied to best match the release of available potential energy observed in the resolved experiment. At first glance, all parameterization schemes similarly represent the large-scale behavior of the eddy- 


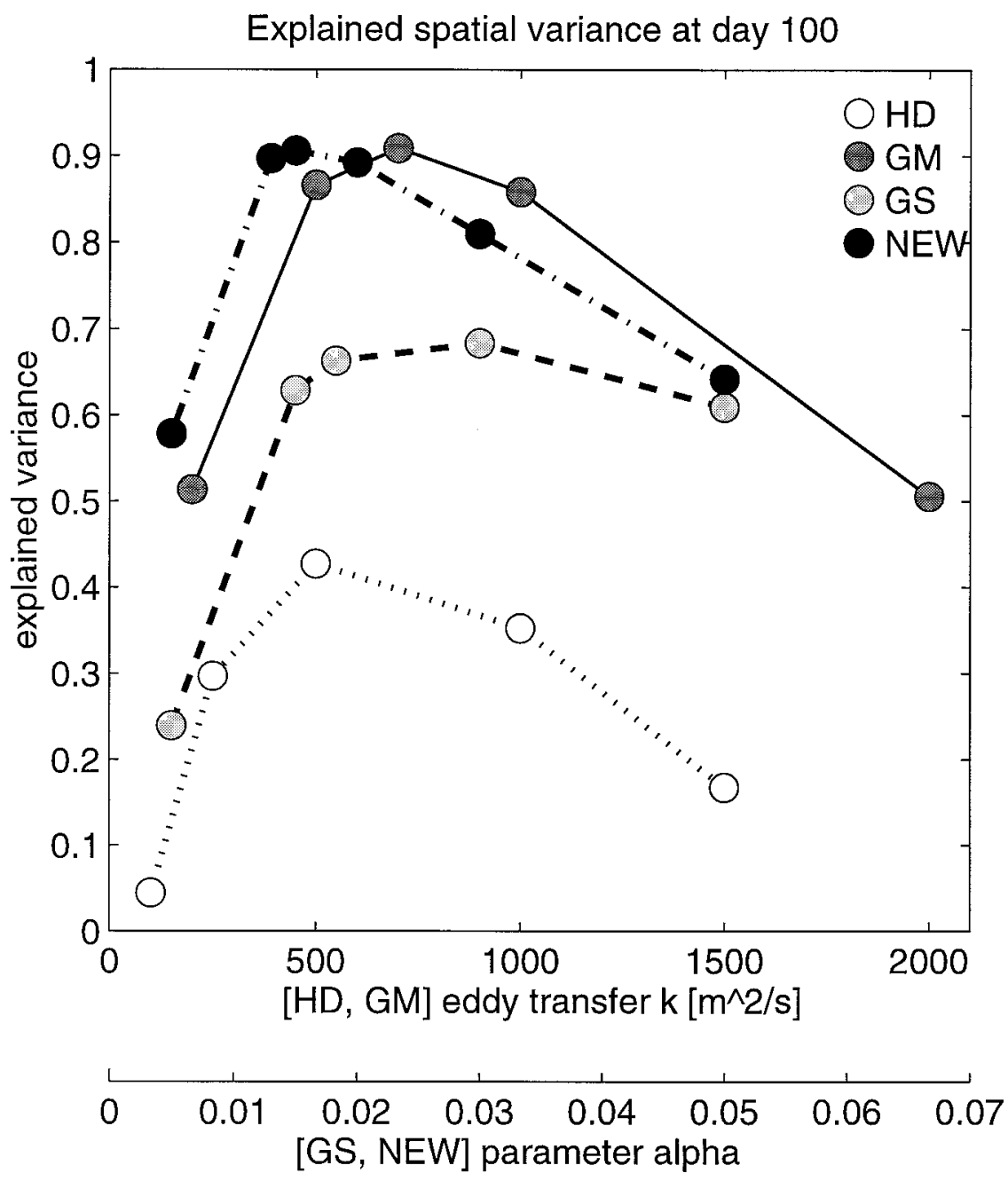

FIG. 10. Explained spatial variance at day 100 of the frontal spindown scenario versus $k$ for HD and GM and versus $\alpha$ for GS and NEW.

resolving model (Fig. 8). However, careful comparison of the volume of fluid between two isopycnal surfaces (e.g., the shaded region in Fig. 8) shows that horizontal diffusion increases the shaded volume with time because of the diapycnal mixing implicit in it.

A measure of the available potential energy is given by the half-domain average density to the north divided by the total mean density (Fig. 9a). As expected, all eddy parameterization schemes were able to more or less collapse the eddy-resolving reference simulation when appropriately tuned.

The total potential energy diagnostic, however, discriminates more clearly between the different schemes (Fig. 9b). Horizontal diffusion does not change the potential energy since fluid is only mixed on horizontal surfaces. The adiabatic GM and NEW schemes, however, lower the center of gravity much as is observed in the eddy-resolving model, the GS scheme somewhat less than observed.

The sensitivity of the various eddy transfer parame- terization schemes with regards to their tunable parameter $(k$ or $\alpha)$ is explored using the explained spatial variance (23) as a measure of their skill (Fig. 10). As expected, the GM and NEW scheme can simulate the adiabatic eddy resolving calculations rather better than the others. However, all schemes show a plateau of acceptable performance centered around the values given in Table 3. Note that the optimum $\alpha$ for the NEW scheme is the same as for the chimney scenario, while the transfer coefficient for the GM scheme had to be increased to $500 \mathrm{~m}^{2} \mathrm{~s}^{-1}$.

\section{c. A wind-driven channel}

The third scenario is wind-driven flow in a periodic channel of width $500 \mathrm{~km}$, length $1500 \mathrm{~km}$, and depth $4500 \mathrm{~m}$. It could be thought of as a model of a narrow ACC. However, it is in the Northern Hemisphere on an $f$ plane with $f=10^{-4} \mathrm{~s}^{-1}$. Stratification of the column increased exponentially to the surface with a top to bot- 
Day : 420

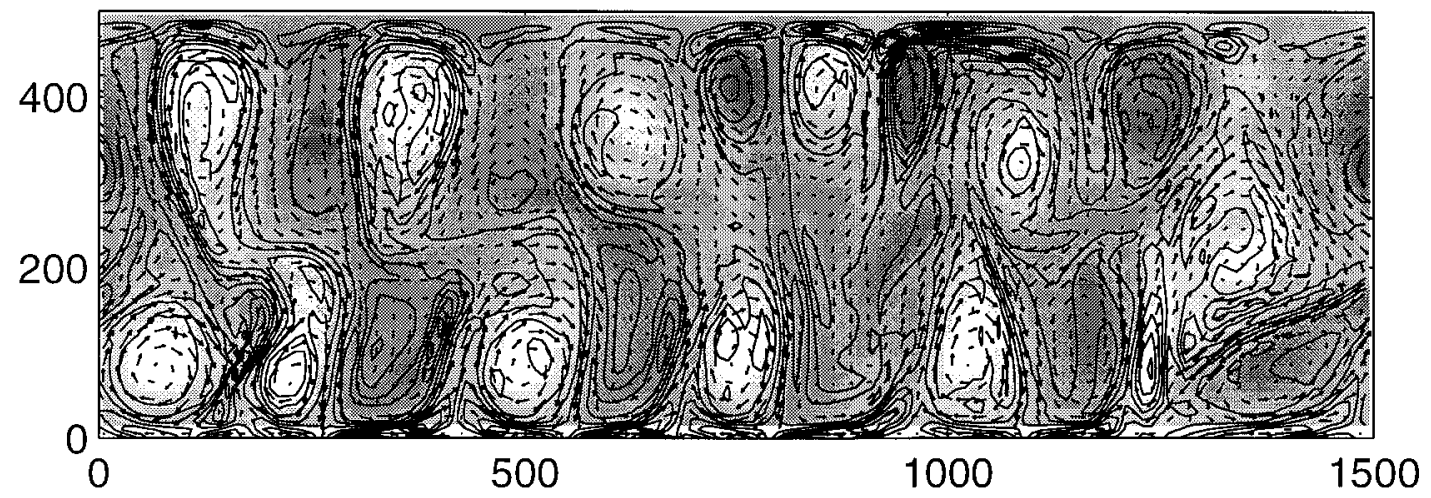

\section{Day : 600}
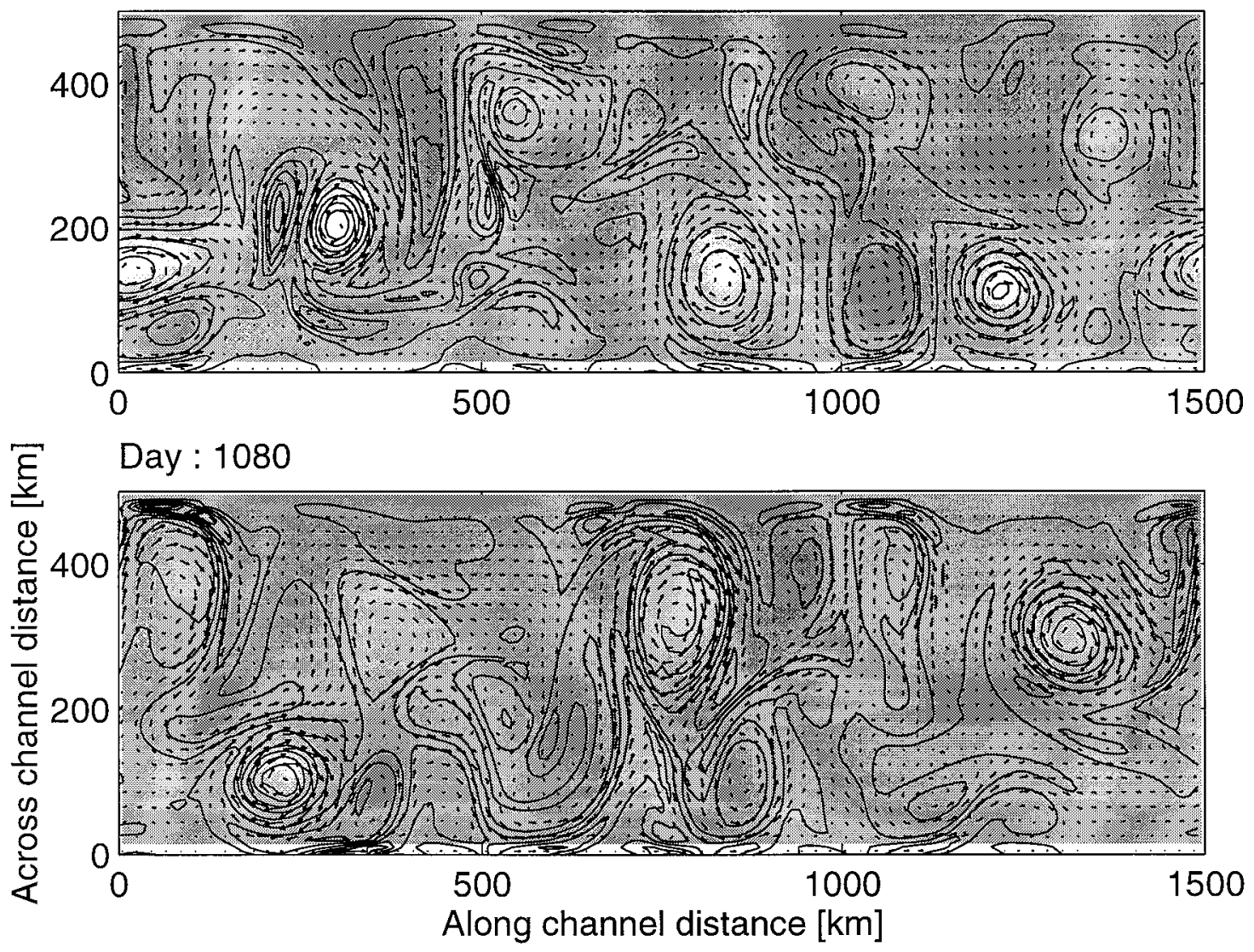

Temperature [C].

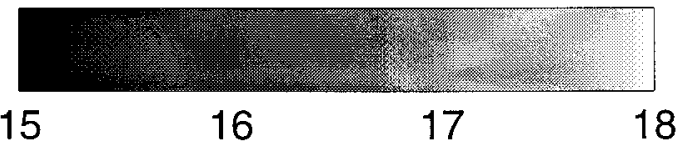

FIG. 11. Surface currents from the eddy-resolving model after 420, 600, and 1080 days. The temperature is shaded with heavy shading denoting denser water. Contours of relative vorticity are shown by solid lines. 

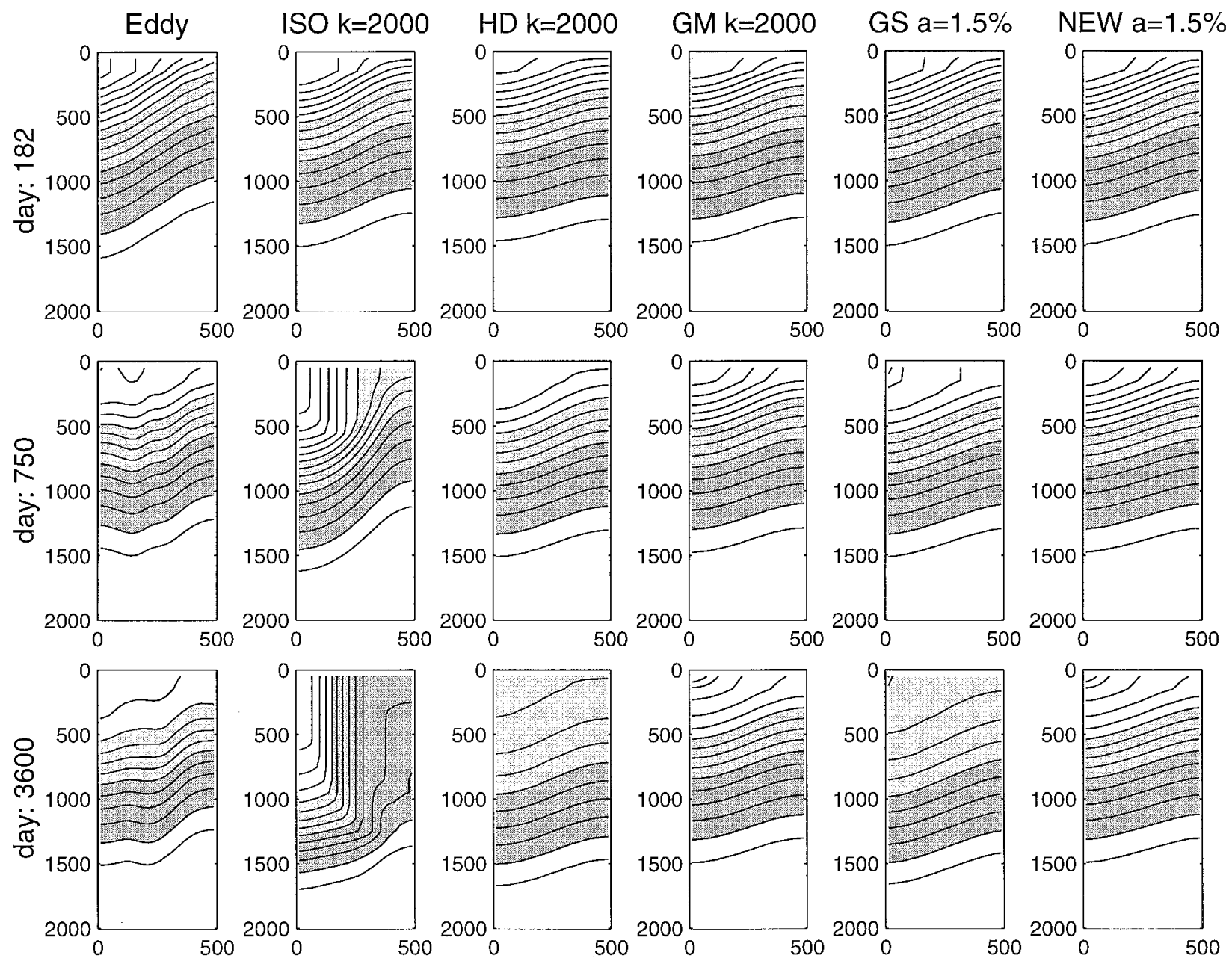

FIG. 12. Density for the wind-driven channel at day 182, 750, and 3600 (10 years) for the eddy-resolving model (left column) and the parameterized 2D model for different parameterization schemes (see text for details). The contour interval is $0.05 \mathrm{~kg} \mathrm{~m}^{-3}$ and two layers are shaded.

tom density gradient of $1 \mathrm{~kg} \mathrm{~m}^{-3}$ and a scale depth of $900 \mathrm{~m}$. The vertical grid spacing was $25 \mathrm{~m}$ at the upper layer increasing to $400 \mathrm{~m}$ at a depth of $4300 \mathrm{~m}$ (Table 1 ). The wind stress had cosine form, decaying to zero at the zonal boundaries and attaining a maximum strength of $0.2 \mathrm{~Pa}$ at the channel center. After about one year isopycnals had been displaced downward by 500 $\mathrm{m}$ or so in the Ekman convergence zone, sufficiently for the flow to become baroclinically unstable. Thereafter a few modes grew to finite amplitude until "Rossby wave breaking" occurred; available potential energy was discharged suddenly (Fig. 11, top panel) and thereafter recovered rather slowly. In the following years eddy formation occurred more irregularly and a broader spectrum of waves facilitated the energy release (Fig. 11, lower panels). Finally, after six years or so, a steady state was reached where the input of potential energy by the wind was balanced by release of potential energy due to baroclinic eddies.

In this quasi-steady state the isopycnals were pushed down vertically typically $250 \mathrm{~m}$ over a horizontal distance of $500 \mathrm{~km}$ (Fig. 12, left column) giving rise to an along-channel jet speed of about $7 \mathrm{~cm} \mathrm{~s}^{-1}$ in the mean; typical eddy speeds were at least twice as large. All eddy flux parameterizations were able to balance the input of available potential energy, as indicated in Fig. $13 a$.

Again major differences arise from the level of diapycnal mixing implicit in the various schemes. Horizontal diffusion, and to a lesser degree the GS scheme, erode the pycnocline significantly, leading to a marked and unrealistically large increase in potential energy with time (Fig. 13b). The potential energy for the GM and NEW runs increased more slowly and very much more in accord with the eddy-resolving experiment.

After 10 years the pycnocline in the center of the channel shows an interesting structure (Fig. 14). Significant diapycnal mixing, in both the HD and GS schemes, warms the central thermocline waters and cools the surface layer. When used in conjunction with 

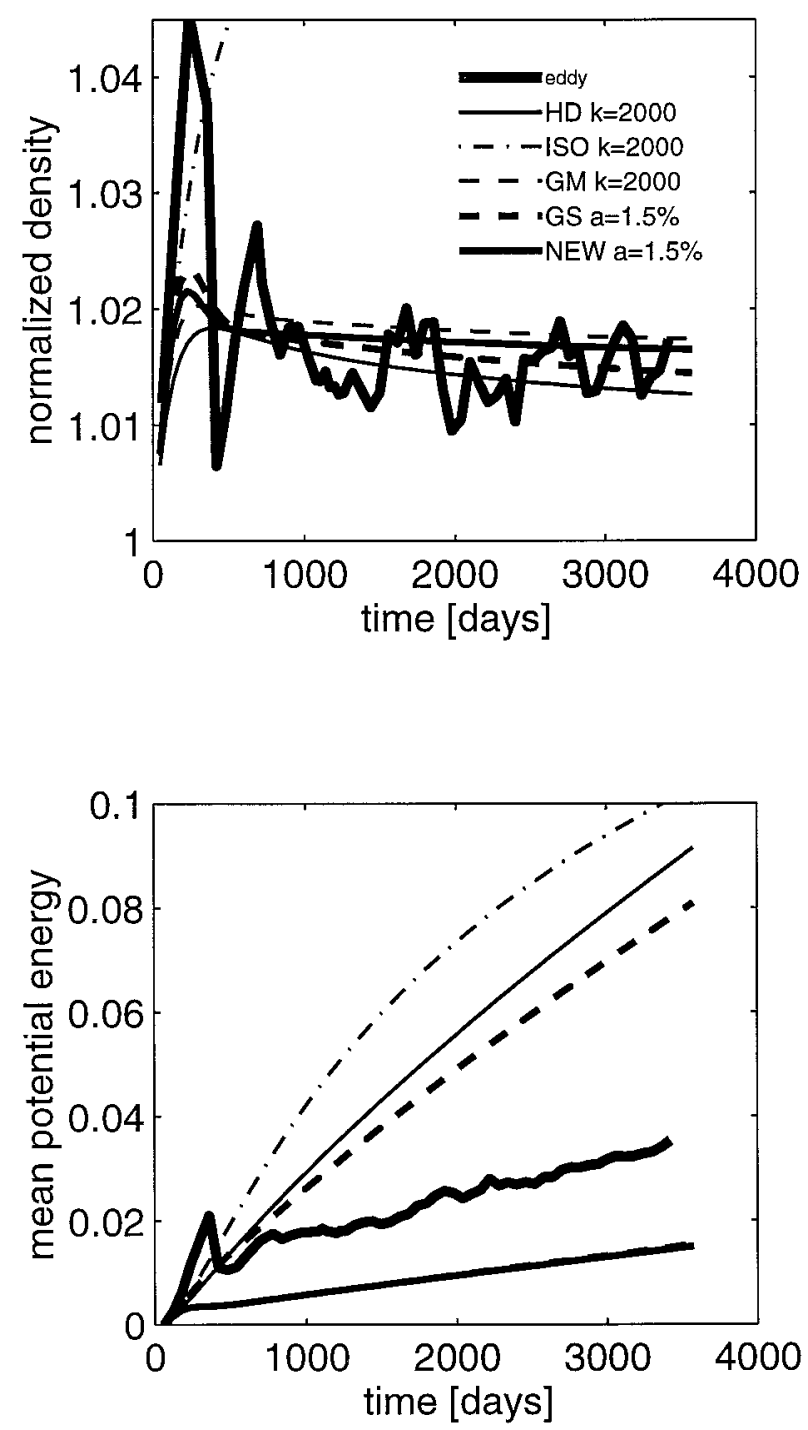

FIG. 13. (a) Evolution of the mean density in the right domain half normalized by the initial density. (b) Evolution of the total potential energy normalized by the initial potential energy.

a surface temperature restoring condition such diapycnal mixing, implicit in the horizontal eddy parameterization, is likely to be responsible for the warming of the thermocline commonly observed in coarse-resolution ocean models (e.g., Danabasoglu et al. 1994).

It should be noted that in some runs convective overturning occurred and was most extreme when the input of available potential energy was not removed parametrically. This is clearly seen in the 2D run ISO, where only diffusion along isopycnal surfaces was present. In this case the Ekman surface flow advected denser water over lighter intermediate water and-after convective adjustment - formed a deep mixed layer (Fig. 14).

The sensitivity of the parameterization schemes was evaluated using the explained variance after 10 years.
However, the diagnostic was confined to levels below 500-m depth (Fig. 15) to focus on the pycnocline of the models. The skills of the parameterized model are not as good as in the previous examples. However, the GM and NEW schemes are clearly the most satisfactory. It is gratifying to observe that the coefficient for the NEW scheme $(\alpha=0.010)$ is very similar to both previous values and earlier studies. The $k$ of the GM scheme, however, had to be adjusted considerably from 300, 500, to $2000 \mathrm{~m}^{2} \mathrm{~s}^{-1}$ before they yielded satisfactory results (Fig. 16, Table 3).

\section{Discussion}

In this paper we have attempted to develop and apply the ideas of Green (1970) and Stone (1972) to improve the parametric representation of baroclinic eddies in large-scale ocean models. Rather than characterize the transfer properties of oceanic eddies by a constant and unchanging transfer coefficient " $k$," we have argued for a $k$ of the form

$$
\begin{aligned}
k & =\alpha \frac{f}{\sqrt{\mathrm{Ri}}} l^{2}=\alpha \frac{M^{2}}{n} l^{2}, \\
\alpha & =0.015 \pm 0.005,
\end{aligned}
$$

where $\alpha$ is a constant of proportionality, $l$ is a measure of the eddy transfer scale, and $M^{2}, N^{2}$ are stratification parameters. By comparing parameterized and resolved experiments in three different oceanic scenarios and assuming that the transfer scale $l$ is that of the width of the baroclinic zone, we have shown that the optimum choice of $\alpha$ is almost constant and equal to 0.015 . The magnitude of the $k$ implied by (26), however, ranges from 300 to $2000 \mathrm{~m}^{2} \mathrm{~s}^{-1}$. Moreover, to within margins of error, it is remarkable that $\alpha$ takes on a similar numerical value to that deduced by Green (1970) (0.005) by fitting his parcel theory of heat transport by atmospheric synoptic-scale systems to atmospheric data. Visbeck et al. (1996) obtained a value of 0.008 in a laboratory and numerical study of isolated convective regions in the ocean. It is noteworthy that Larichev and Held (1995) arrive at the form (26) but from the rather different perspective of homogeneous geostrophic turbulence calculations.

An important aspect of (26) is that it ascribes physical attributes to $k$ and therefore a context for enquiry and further refinement of a transfer scale $l$, a timescale associated with baroclinic eddies $M^{2} / N$, and a proportionality constant $\alpha$ that measures the efficiency of the transfer process. A deeper understanding of those processes that determine these key factors (see Larichev and Held 1995 ) is required before a more complete representation of $k$ can be deduced.

Our comparison of resolved and parameterized models clearly shows that the transformed Eulerian mean representation advocated by Gent and McWilliams (1990) offers advantages over more conventional ap- 


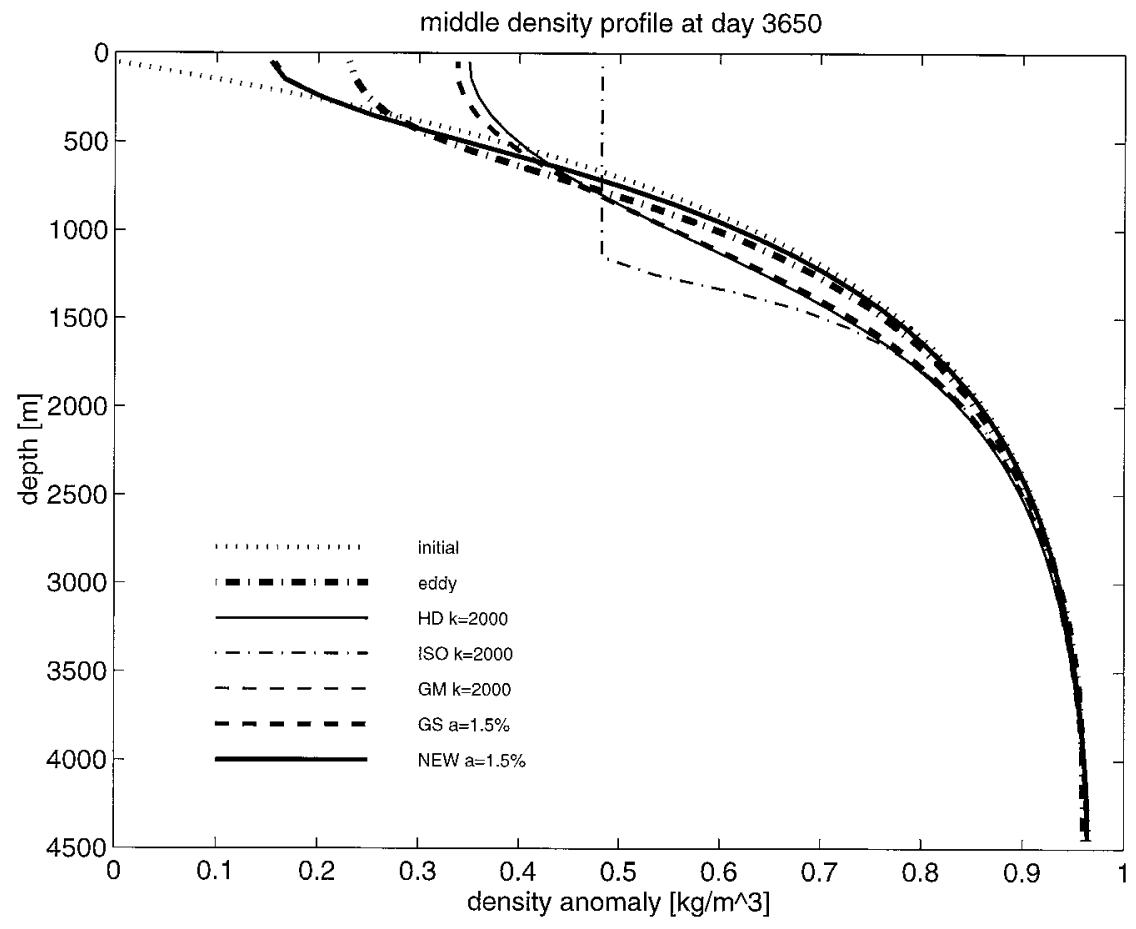

FIG. 14. Density profiles in the center of the channel after 10 years of integration for different parameterization, the eddy-resolving model, and the initial condition.

proaches. Although Green and Stone provide a way forward in the prescription of the $k$, their adoption of a "mixing" formalism of the kind $\boldsymbol{\nabla} \cdot(k \boldsymbol{\nabla} T)$ leads to clear problems when compared with explicit models. We therefore argue for a representation of baroclinic eddies that combine the best aspects of GS and GM: transfer coefficients that vary in space and time according to (26), together with the adoption of a transformed Eulerian mean formalism.

We have restricted our attention to problems where the rotation rate is constant ( $f$ plane) and, hence, the lateral growth of fronts is only limited by the domain size. However, if the rate of rotation varies significantly within the domain of interest, an upper bound on the mixing length scale $l$ will appear at a scale given by $l_{\beta}$ $\sim \sqrt{U / \beta}$, which separates a turbulent from a wavelike regime (Rhines 1975, 1977). The importance of planetary $\beta$ and topographic $\beta$ as limiting factors on eddy transfer is deferred to future research.

We have focused our attention on three problems that possess a strong degree of symmetry in the alongfront direction and for which baroclinic instability is the dominant mechanism of lateral mixing. In the more general context of the oceanic general circulation the mean flow may be spatially inhomogeneous in all three dimensions, and thus may contain regions in which the eddy fluxes are not governed by simple local stability theory. Nonlocal influences may arise from the local generation of wave activity and its subsequent advection and radiation to the far field (Holland and Rhines 1980). Eddy variability is also generated by barotropic instabilities, local surface or topographic forcing, and through wave radiation from distant forcing regions. Additional parameterizations will certainly be necessary to represent the eddy mean flow interaction in such an inhomogeneous environment.

Thus, while the present set of examples provides a clean and simple basis for evaluation of the various eddy flux parameterizations discussed here, it is clear that additional factors are likely to become important in moving to the problem of parameterizing eddy fluxes in the general oceanic circulation where many dynamical regimes are present.

Finally, in this discussion we have not addressed the role and parameterization of momentum transport by baroclinic eddies. To make progress here we believe that one must couch the transfer process in terms of the quasi-conserved potential vorticity (see Marshall 1981) rather than heat and momentum separately.

Plans to implement and evaluate the ideas set out here in a general circulation model are underway and will be reported later.

Acknowledgments. We would like to thank M. Follows, D. Marshall, J. Marotzke, P. Stone, and T. McDougall for helpful discussions. Two anonymous reviewers and J. Pedlosky provided useful suggestions to improve the manuscript. MV was funded by a UCAR/ 

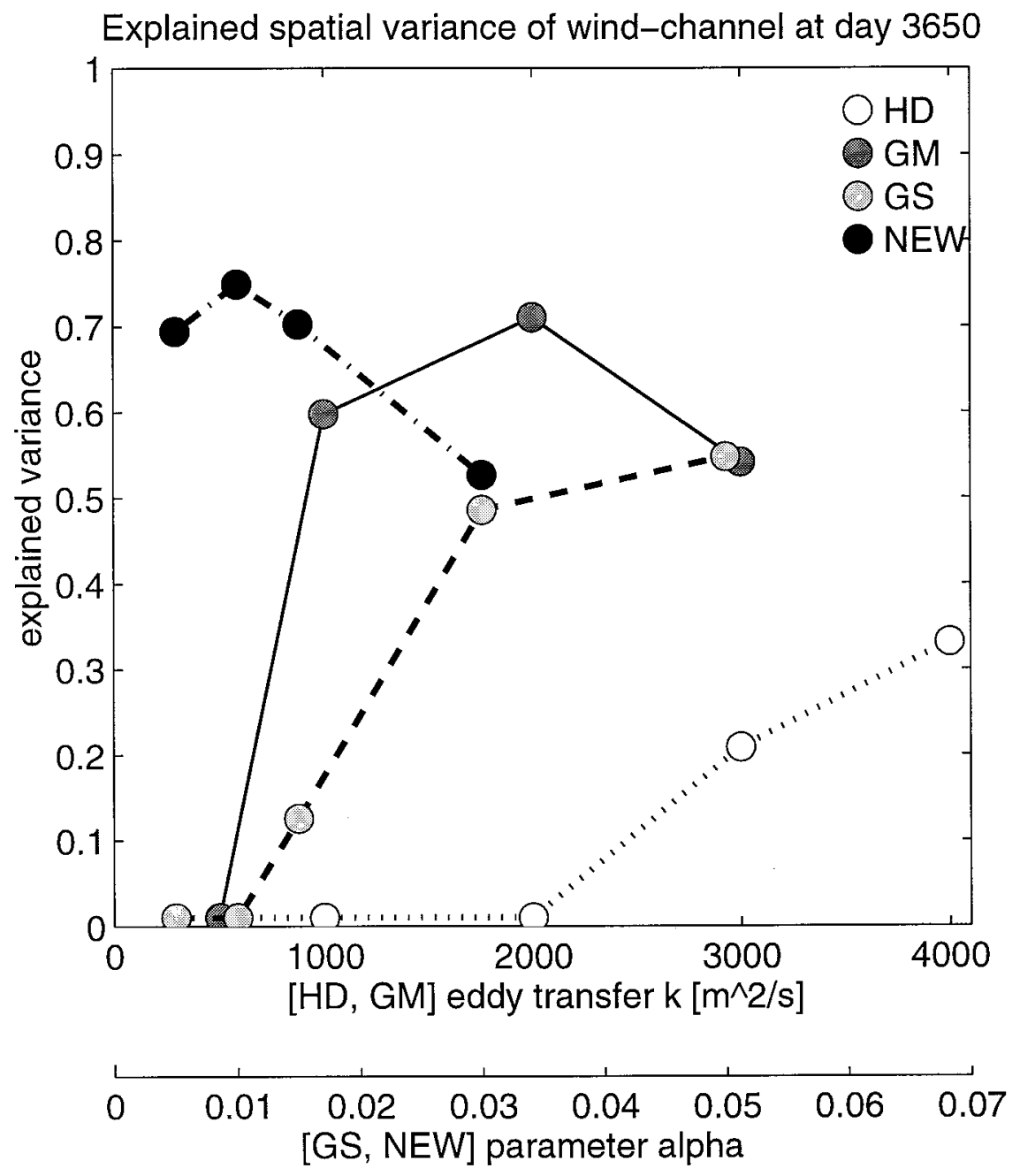

FIG. 15. Explained spatial variance referenced to the eddy-resolving model field after 10 years below 500-m depth for the different parameterization versus $k$ for HD and GM and versus $\alpha$ for GS and NEW.

NOAA fellowship on Global and Climate Change. JM was supported by ONR and NSF, and TH by NOAA ACCP. MS was supported by ONR Grant N00014-93$1-0572$.

\section{APPENDIX}

\section{The Two-Dimensional Model}

We employ a two-dimensional model to test the various eddy transfer parameterization schemes. The 2D model has linearized momentum equations assuming a balanced flow at a small Rossby number in the Boussinesq and hydrostatic limit and thereby neglect the Reynolds stress terms. We arrive at the following diagnostic set of momentum equations for the mean properties:

$$
\begin{aligned}
-f v & =-r u+T_{x} \\
f u & =-\frac{1}{\rho_{0}} \frac{\partial p}{\partial y}-r v+T_{y} \\
\frac{g \rho}{\rho_{0}} & =-\frac{1}{\rho_{0}} \frac{\partial p}{\partial z},
\end{aligned}
$$

where $f$ is the Coriolis parameter, which is assumed constant ( $f$ plane), and the zonal pressure gradient has been set to zero. Friction is represented as a body force $\left(r=r_{i}+r_{b}(z)\right)$ comprising an interior Newtonian damping coefficient $r_{i}$ plus bottom drag $r_{b}\left(u_{b}, v_{b}\right) ; T_{x}$ and $T_{y}$ represent wind stress in the surface layer.

The continuity equation is

$$
\frac{\partial v}{\partial y}+\frac{\partial w}{\partial z}=0
$$



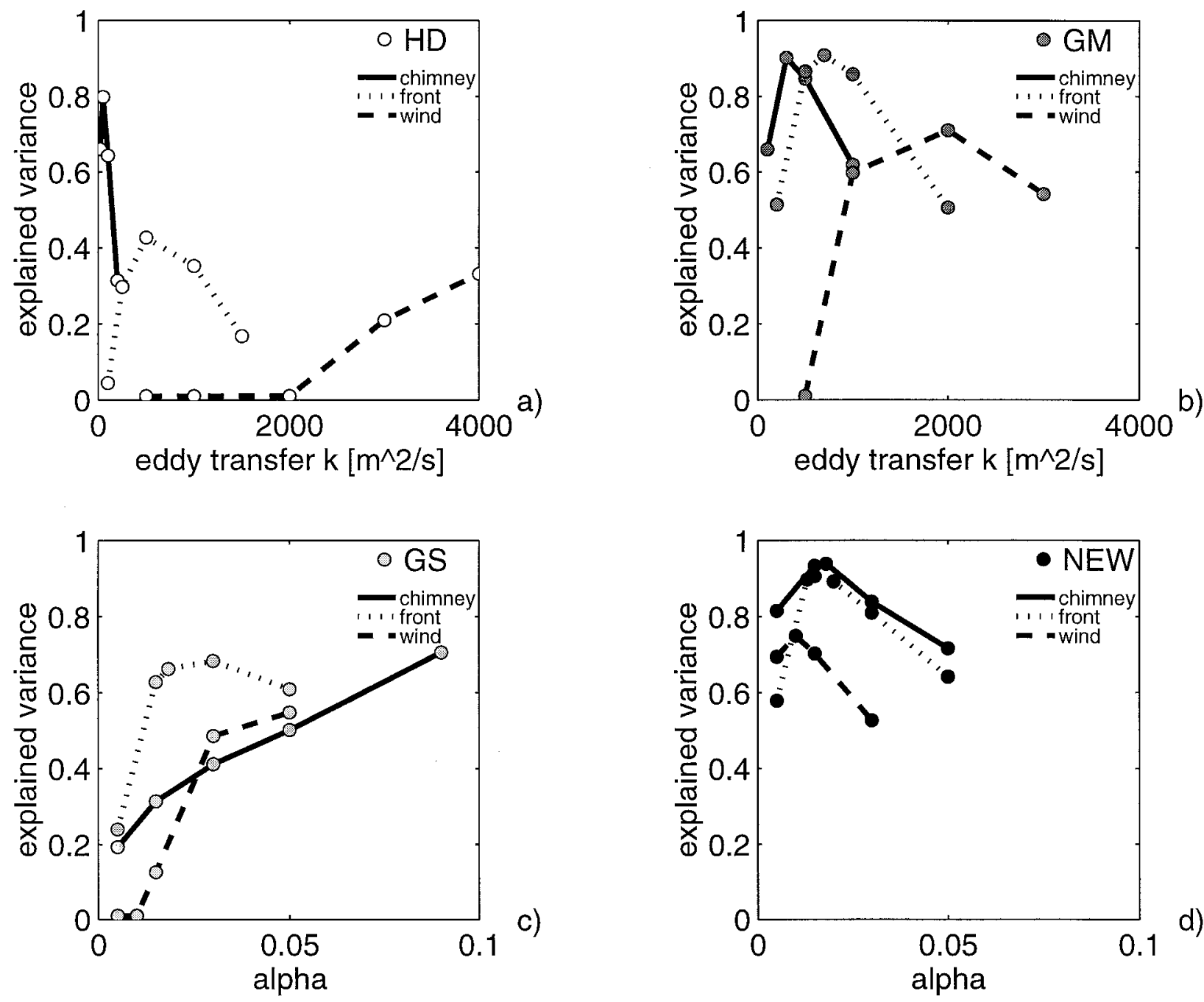

FIG. 16. Explained spatial variance referenced to the eddy-resolving model calculations for all scenarios and the four parameterizations.

yielding a diagnostic equation for the vertical velocity $w$. The tracer equations for temperature, salinity, and passive tracer are a prognostic, fully nonlinear two-dimensional version of (3):

$$
\frac{\partial \tau}{\partial t}+v \frac{\partial \tau}{\partial y}+w \frac{\partial \tau}{\partial z}=S_{0}-\nabla_{\mathbf{h}} \cdot \mathbf{F},
$$

where $S_{0}$ denotes sources and sinks of tracer concentration and

$$
\mathbf{F}=\left(F_{y}, F_{z}\right)=\left(\overline{v^{\prime} \tau^{\prime}}, \overline{w^{\prime} \tau^{\prime}}\right)
$$

represents the subgrid eddy transfer (where the prime denotes the departure from a zonal average). To obtain a zonally averaged closure for (A5), the eddy flux must be parameterized in terms of zonally averaged quantities. We proceed by means of a flux-gradient relation

$$
\mathbf{F}=-\mathbf{K} \cdot \nabla_{h} \tau_{n}
$$

where $\tau_{n}$ is the zonally averaged tracer concentration, and $\mathbf{K}$ is a tensor, which accommodates the subgridscale transfer schemes discussed in sections 2 and 3.

Finally, an equation of state is needed that relates the density to the tracer fields:

$$
\rho=\sum_{n}\left(\frac{\partial \rho}{\partial \tau_{n}}\right) \tau_{n}
$$

where the expansion coefficients $(\partial \rho / \partial \tau)$ can be nonlinear functions.

\section{a. Method of solution}

The 2D model equations for the variables $(u, v, w, p$, $\left.\tau_{1} \ldots, \tau_{n}, \rho\right)$ are solved numerically on a $\mathrm{C}$ grid using a leapfrog time-stepping scheme and a Smolarkiewicz (1983) advection scheme. We employ a rigid lid at the surface and a no flux condition normal to the boundaries.

The horizontal momentum equations (A1), (A2) can 
be combined to obtain the following diagnostic equation for the cross-channel velocity:

$$
v=\left(\frac{r}{r^{2}+f^{2}}\right)\left(T_{y}-\frac{1}{\rho_{0}} \frac{\partial p}{\partial y}\right)-\left(\frac{f}{r^{2}+f^{2}}\right) T_{x} .
$$

The along-channel velocity

$$
u=\left(\frac{f}{r^{2}+f^{2}}\right)\left(T_{y}-\frac{1}{\rho_{0}} \frac{\partial p}{\partial y}\right)+\left(\frac{r}{r^{2}+f^{2}}\right) T_{x}
$$

need not to be computed in the prognostic integration. The character of the solution depends on the ratio $r / f$. If $r>f$ and therefore $r^{2} \gg f^{2}$, we obtain a frictional solution with flow down the meridional pressure gradient. If $f>r$, the dynamics are rotationally controlled with $u$ in geostrophic balance and only small ageostrophic flow in the $y-z$ plane. The latter regime is of interest for large-scale oceanic flows and we typically choose $r / f<10^{-2}$.

To solve for the surface pressure gradient, the full pressure $p$ is split into the surface pressure $p_{s}$ and the internal hydrostatic pressure $p_{i}$ such that

$$
p=p_{i}+p_{s}, \quad p_{i}=-\int_{-H}^{0} g \rho d z
$$

and $p_{i}$ is obtained by vertically integrating the hydrostatic equation (A3). Integrating the continuity equation (A4) vertically yields

$$
\int_{-H}^{0} \frac{\partial v}{\partial y} d z=0
$$

and differentiating (A9) with respect to $y$ and splitting the velocities into an internal $v_{i}$ and the barotropic part $v_{s}$ gives

$$
\frac{\partial v}{\partial y}=\frac{\partial\left(v_{s}+v_{i}\right)}{\partial y}=-\frac{r}{\rho_{0}\left(f^{2}+r^{2}\right)} \frac{\partial^{2} p_{s}}{\partial y^{2}}+\frac{\partial v_{i}}{\partial y} .
$$

Together with (A12) the following equation for curvature of the surface pressure results:

$$
\frac{\partial^{2} p_{s}}{\partial y^{2}}=\left(\int_{-H}^{0} \frac{r}{\rho_{0}\left(f^{2}+r^{2}\right)} d z\right)^{-1} \int_{-H}^{0} \frac{\partial v_{i}}{\partial y} d z
$$

Equation (A14) is then solved to obtain the surface pressure gradient with the imposition of one further boundary condition, zero surface pressure gradient at one boundary.

\section{b. Transfer coefficients}

The transfer coefficients for the GS and NEW schemes were computed at each time step using the method described in section $3 \mathrm{~d}$. However, the $k$ 's need not be evaluated so frequently, and we compute them at a rate equivalent to an Eady growth rate $t_{e}=f / \sqrt{\mathrm{Ri}}$ $=M^{2} / N$.

To allow for a smooth time evolution of the transfer coefficients, a characteristic timescale $T_{\text {relax }}$ is introduced and the $k$ are filtered, as follows:

$$
k_{\text {new }}=k_{\text {old }}+\frac{\Delta t}{T_{\text {relax }}}\left(k-k_{\text {old }}\right),
$$

where $\Delta t$ denotes the model time step. It is possible to interpret the relaxation time $\left(T_{\text {relax }}\right)$ in terms of an eddy growth rate, and one might use $t_{e}$ as the relaxation time.

\section{REFERENCES}

Andrews, D., and M. McIntyre, 1976: Planetary waves in horizontal and vertical shear: The generalized Eliassen-Palm relation and the mean zonal acceleration. J. Atmos. Sci., 33, 2031-2048.

_- J. Holton, and C. Leovy, 1987: Middle Atmosphere Dynamics. Academic Press, 489 pp.

Bleck, R., and D. Boudra, 1986: Wind-driven spin-up in eddy-resolving ocean models formulated in isopycnic and isobaric coordinates. J. Geophys. Res., 91, 7611-7621.

Danabasoglu, G., J. McWilliams, and P. Gent, 1994: The role of mesoscale tracer transports in the global ocean circulation. Science, 264, 1123-1126.

Eady, E., 1949: Long waves and cyclone waves. Tellus, 1(3), 33-52.

Gent, P., and J. McWilliams, 1990: Isopycnal mixing in ocean circulation models. J. Phys. Oceanogr., 20, 150-155.

—_, J. Willebrand, T. McDougall, and J. McWilliams, 1995: Parameterizing eddy-induced tracer transports in ocean circulation models. J. Phys. Oceanogr., 25, 463-474.

Green, J. S., 1970: Transfer properties of the large-scale eddies and the general circulation of the atmosphere. Quart. J. Roy. Meteor. Soc., 96, 157-185.

Haine, T., and J. Marshall, 1997: Gravitational, symmetric and baroclinic instability in the ocean mixed layer. J. Phys. Oceanogr.,

Holland, W., and P. Rhines, 1980: An example of eddy-induced ocean circulation. J. Phys. Oceanogr., 10, 1010-1031.

Jones, H., and J. Marshall, 1993: Convection with rotation in a neutral ocean: A study of open-ocean deep convection. J. Phys. Oceanogr., 23, 1009-1039.

Large, W., J. McWilliams, and S. Doney, 1994: Oceanic vertical mixing: A review and a model with a nonlocal boundary layer parameterization. Rev. Geophys., 32, 336-403.

Larichev, V., and I. Held, 1995: Eddy amplitudes and fluxes in a homogeneous model of fully developed baroclinic instability. $J$. Phys. Oceanogr., 25, 2285-2297.

Marshall, J., 1981: On the parameterization of geostrophic eddies in the ocean. J. Phys. Oceanogr., 11, 257-271.

_- and G. Shutts, 1981: A note on rotational and divergent eddy fluxes. J. Phys. Oceanogr., 11, 1677-1680.

_ _ A. Adcroft, C. Hill, L. Perelman, and C. Heisey, 1996a: A finite volume, incompressible Navier-Stokes model for studies of the ocean on parallel computers. J. Geophys. Res., in press.

_ C. Hill, L. Perelman, and A. Adcroft, 1996b: Hydrostatic, quasihydrostatic and non-hydrostatic ocean modeling. J. Geophys. Res., in press.

Maxworthy, T., and S. Narimousa, 1994: Unsteady, turbulent convection into a homogeneous, rotating fluid, with oceanographic applications. J. Phys. Oceanogr., 24, 865-887.

McDougall, T., and P. McIntosh, 1996: The temporal-residual-mean velocity. Part I: Derivation and the scalar conservation equations. J. Phys. Oceanogr., 26, 2653-2665.

McWilliams, J., and P. Gent, 1994: The wind-driven ocean circulation with an isopycnal-thickness mixing parameterization. J. Phys. Oceanogr., 24, 46-65.

Plumb, R., and J. Mahlman, 1987: The zonally averaged transport characteristics of the GFDL general circulation/transport model. J. Atmos. Sci., 44, 298-327.

Redi, M., 1982: Oceanic isopycnal mixing by coordinate rotation. $J$. Phys. Oceanogr., 12, 1154-1157. 
Rhines, P., 1975: Waves and turbulence on the $\beta$-plane. J. Fluid Mech., 69, 417-443.

_- 1977: The dynamics of unsteady currents. The Sea, Vol. 6, E. Goldberg, Ed., Wiley, 189-318.

- and W. Young, 1982: Homogenization of potential vorticity in planetary gyres. J. Fluid Mech., 122, 347-368.

Saltzman, B., 1978: A survey of statistical-dynamical models of the terrestrial climate. Advances in Geophysics, Vol. 20, Academic Press, 183-204.

Smolarkiewicz, P., 1983: A simple positive definite advection scheme with small implicit diffusion. Mon. Wea. Rev., 111, 479-485.

Solomon, H., 1971: On the representation of isentropic mixing in ocean circulation models. J. Phys. Oceanogr., 1, 233-234.

Spall, M., 1995: Frontogenesis, subduction, and cross-front exchange at upper ocean fronts. J. Geophys. Res., 100, 2543-2558.

Stone, P., 1972: A simplified radiative-dynamical model for the static stability of rotating atmospheres. J. Atmos. Sci, 29, 405-418.

_, and M.-S. Yao, 1990: Development of a two-dimensional zo- nally averaged statistical-dynamical model. Part III: The parameterization of the eddy fluxes of heat and moisture. J. Climate, 3, 726-740.

Treguier, A., I. Held, and V. Larichev, 1997: On the parameterization of quasigeostrophic eddies in primitive equation ocean models. J. Phys. Oceanogr., in press.

Turner, J., 1973: Buoyancy Effects in Fluids. Cambridge University Press.

Visbeck, M., J. Marshall, and H. Jones, 1996: Dynamics of isolated convective regions in the ocean. J. Phys. Oceanogr., 26, 17211734.

White, A. A., and J. S. A. Green, 1984: Transfer coefficient eddy flux parameterizations in a simple model of the zonal average atmospheric circulation. Quart. J. Roy. Meteor. Soc., 110, 10351052.

Whitehead, J., J. Marshall, and G. Hufford, 1996: Localized convection in rotating stratified fluid. J. Geophys. Res., 101, $25705-$ 25722 . 